\title{
4. Zur wissenschaftlichen Arbeit der Anstalt
}

\subsection{Allgemeine Bemerkungen}

Der Rahmen für die wissenschaftliche Arbeit der Anstalt war bereits 1876 in der "Denkschrift betreffend die Errichtung einer biologischen Anstalt auf Helgoland" festgelegt worden. Schwerpunkt der geplanten Forschungsarbeit sollten alle Fragen sein, die mit der Fischerei zusammenhängen. Sie wurden im Abschnitt „Aufgaben der reinen Biologie“ so zusammengefaßt:

- die Erforschung der Fischgründe der Nordsee auf ihre Bodenbeschaffenheit, ihre Tierwelt und die Produktion an Nutzfischen;

- systematische Versuche mit der künstlichen Aufzucht von Nutzfischen (einschließlich Hummern);

- Ermittlungen zur Festsetzung der Schonmaßregeln und Schonzeiten für Nutzfische;

- monographische Bearbeitung der wichtigsten Nutzfische der deutschen Meere (Hering, Aal, Schellfisch, Scholle usw.), namentlich ihre Entwicklung, Ernährung und Wanderungen.

Als allgemeine Aufgaben wurden genannt:

- die Erforschung der ganzen Nordsee, insbesondere der Meeresumgebung von Helgoland nach der physikalisch-chemischen, geologischen und zoologisch-botanischen Seite hin;

- die Untersuchung besonders wichtiger Erscheinungen im Leben des Meeres wie Meeresleuchten, das Auftreten größerer Schwärme usw.;

- die Erforschung des Plankton als Urproduktion des Meeres;

- die Erforschung der Geologie, der Landfauna und Landflora von Helgoland.

Dieses Programm war sehr umfangreich und nicht alle Vorhaben konnten von den wenigen Mitarbeitern mit gleicher Intensität bearbeitet werden. Schwerpunkt war die Fischereibiologie. Auf diesem Gebiet konnte an beträchtliche Vorleistungen von Mitarbeitern angeknüpft werden.

So hatte Heincke bis 1892 über dreißig Arbeiten vorgelegt (vgl. Ehrenbaum 1922), von denen sich mehrere mit dem Hering (Clupea harengus) befaßten. Auch Ehrenbaum konnte bereits auf eine Vielzahl von Arbeiten verweisen, die sich sowohl mit der praktischen Fischerei als auch mit zoologischen Themen im engeren Sinne befaßten (vgl. Lüling 1949). An ihre Ergebnisse konnten spätere Mitarbeiter der Anstalt anschließen. Anders war es mit den Arbeiten zur Planktonforschung. Hier gab es zu Anfang große Schwierigkeiten, die von Hensen ausgearbeiteten Methoden anzuwenden. Auch die im Programm angeführten geologischen Arbeiten spielten kaum eine Rolle und beschränkten sich auf Erdbebenmessungen, Im Keller des Anstaltsgebäudes ,Kaiser von Deutschland“ befand sich eine Erdbebenstation, die vom Präparator der Station betreut wurde, außerdem war die Anstalt für eine meteorologische Station verantwortlich.

Die Umsetzung des Forschungsprogramms bzw. seine Erweiterung war auf Helgoland nicht nur von den technischen Möglichkeiten abhängig, sondern in besonderem Maße von den angestellten Mitarbeitern und ihrer Initiative. Paul Kuckuck, ab 1892 als Botaniker aus Mitteln beschäftigt, die die Königlich-Preußische Akademie der Wissenschaften zur Verfügung stellte, führte Arbeiten zur Biologie der Algen durch und begründete damit die botanische Tradition der Anstalt. Das Verdienst Hugo Weigolds war es, ab 1910 die ornithologische Forschung an der Anstalt eingeführt zu haben. Physiologisch wurde an der Anstalt 
erstmals von Viktor Franz zwischen 1908 und 1910 gearbeitet, die Forschungen erreichten einen Aufschwung mit der Verbesserung der technischen Möglichkeiten im neuen Gebäude.

Die Arbeiten auf dem Gebiet der Fischereizoologie wurden stets dominiert von wirtschaftlichen Zwängen, was besonders deutlich während und nach dem I. Weltkrieg und im II. Weltkrieg zum Ausdruck kam. Hier wurde das Forschungsprofil stark eingeengt. So wurden z. B. die botanischen Arbeiten eingestellt. Der Fischereiforschung, die für die Ernährung der Bevölkerung wichtig war, wurde Vorrang eingeräumt.

Verallgemeinernd läßt sich sagen, daß zwischen 1892 und 1945 auf folgenden Gebieten gearbeitet wurde:

- Forschungen zur marinen Biologie (Forschungen zur Systematik, Zoologie, Meeresbotanik);

- Arbeiten im Dienste der internationalen Meeresforschung (Arbeiten zur Systematik und Entwicklungsgeschichte von Meerestieren als Voraussetzung für gezielten Schutz von Fischbeständen, Planktonforschung, Hydrologie, Geologie);

- Ornithologie (insbesondere ab 1902);

- Physiologie von Meerestieren (ab 1910, verstärkt ab 1921).

Es sollen im folgenden einige Angaben auf der Grundlage von Archivmaterial, vor allem der Berichte des Direktors, präzisiert werden. Eine detaillierte Analyse der wissenschaftlichen Veröffentlichungen der Mitarbeiter der Anstalt möge späterer Arbeit von Fachleuten vorbehalten sein.

\subsection{Zu Forschungen auf ausgewählten Gebieten der marinen Biologie}

In den ersten zehn Jahren des Bestehens der Anstalt, etwa 1892-1902, bemühte man sich, Aufbau und Entwicklung der marinen Lebewesen um Helgoland zu erforschen und die verschiedenen Gruppen mit der Vielfalt ihrer Formen voneinander abzugrenzen. Dabei stand das Bedürfnis im Vordergrund, Fauna und Flora der deutschen Heimat besser kennenzulernen. Dies betraf sowohl die deutschen Meere insgesamt als auch den „zurückgewonnenen" Raum um Helgoland. Man knüpfte an Arbeiten der älteren Forschergeneration an. Neben morphologischer, entwicklungsgeschichtlicher und systematisch-faunistischer Erforschung der Tierwelt galt es besonders, Lücken in der Lebenskunde mariner Nutztiere auszufüllen.

Die ersten Untersuchungen von August bis Oktober 1892 ergaben, daß der Reichtum der Fauna bei Helgoland weit größer war, als man bisher angenommen hatte. Heincke stellte in diesem Zusammenhang fest, daß inzwischen Stoff für die mannigfaltigsten und umfassendsten Arbeiten gefunden sei. Er hob hervor, daß das Untersuchungsmaterial auf Grund der günstigen Lage der Insel gut zugänglich ist. Allein die Zahl der Molluskenarten (Muscheln, Schnecken, Tintenfische), von denen bis 1890 etwa 100 bekannt waren, betrug nach Angaben von Heincke im Jahre 1893 bereits 150.

Er zog daraus in einer "Denkschrift über die Wirksamkeit der Biologischen Anstalt" die Schlußfolgerung, daß die Insel als Standort für die Forschung vorzüglich geeignet sei (8). Hintergrund für diesen rechtfertigenden, Nachweis" waren vermutlich die langjährigen engagierten Diskussionen über den Forschungsstandort Helgoland. Wie aus dem Jahresbericht 1893 des Direktors hervorgeht, waren die ersten Arbeiten der Institutsmitarbeiter Abhandlungen zu Systematik und Lebenskunde von Meeresorganismen. Von Anfang an nahm die Beobachtung der lebenden Organismen einen besonderen Platz ein. Dabei 
konzentrierte man sich auf die nähere Umgebung der Insel. Dies entsprach einem wissenschaftlichen Bedürfnis, aber auch den technischen Möglichkeiten, die zunächst nur kurze Fahrten zuließen. Da nur zwei sehr kleine Boote zur Verfügung standen, war der Untersuchungsradius gering.

Friedrich Heincke arbeitete über Fische und Weichtiere (Mollusca, s.o.), Clemens Hartlaub über Einzeller (Protozoa), Hohltiere (Coelenterata darunter Hydrozoa), Stachelhäuter (Echinodermata), Moostierchen (Bryozoa) und Würmer. Seine Forschung an den Hydrozoa, einer Klasse der Nesseltiere (Cnidaria), die zum einen auf der Beobachtung freilebender Tiere, zum anderen auf Züchtung beruhte, machte ihn international bekannt. So wurden von Hartlaub in den Jahren 1907-10 mehrere Veröffentlichungen zu Medusen vorgelegt (63). Seine Arbeiten über Hydrozoa der nordischen Meere setzte Hartlaub bis zum Beginn des I. Weltkrieges fort. Sie wurden in deutschen wie auch in französischen Zeitschriften veröffentlicht. Nach Einschätzung des späteren Direktors der Anstalt, Adolf Bückmann, stellen sie wichtige Ergebnisse über die Entwicklungsgeschichte und Fortschritte in der Erkenntnis der Artzusammengehörigkeit von Medusen und Polypen dar. Sie waren damit auch von großer Bedeutung für die Systematik dieser Gruppe, die durch das Vorhandensein der beiden getrennten Generationen der Meduse und des Polypen erschwert wird (vgl. Bückmann 1959).

Auch Ernst Ehrenbaum arbeitete zunächst systematisch-morphologisch, z. B. über Krebse (Crustacea) und Ringelwürmer (Annelida), bevor er sich mit Heincke zusammen fischereizoologischen Themen zuwandte. Daran wird die Vernetzung der Forschungsgebiete allgemeine Zoologie, Fischereizoologie und Planktonforschung deutlich. So gingen erste monographische Behandlungen verschiedener Tiergruppen (z. B. durch Hartlaub) wie auch die Arbeiten Ehrenbaums über Eier und Larven von Fischen in das den ganzen Nordatlantik berücksichtigende Bestimmungswerk „Nordisches Plankton“ ein (vgl. Ehrenbaum 1908).

Die zoologischen Arbeiten wurden wesentlich von der Deutschen Wissenschaftlichen Kommission für Meeresforschung unterstützt. Zu den wenigen Themen, die nicht aus Mitteln der Kommission finanziert wurden, gehören physiologisch-chemische Untersuchungen, die von Viktor Franz 1910 begonnen wurden. Hertling und Gebhardt bauten darauf auf, Hertling führte chemisch-physiologische Untersuchungen an Schnecken (Prosobranchia) während ihrer Entwicklung durch, Gebhardt untersuchte marine Ruderfußkrebse (Copepoda). Er versuchte, die Ursache der Färbung aufzuklären, und stellte u. a. Untersuchungen über das Schmeck- und Riechvermögen der Fische an. Ihn interessierten dabei der Einfluß von Licht, thermischen und chemischen Unterschieden im Medium und der Schwerkraft. Physiologische Untersuchungen wurden später von Helmuth Hertling weitergeführt.

Auch die Arbeiten an der Auster (Ostrea edulis) hatten über ihren Grundlagencharakter hinaus (Lebensgemeinschaftsforschung) eine praktische Zielrichtung. Arthur Hagmeier begann seine Untersuchungen bereits vor dem I. Weltkrieg. Er hatte an regelmäßigen Fangfahrten der Austerngesellschaft teilgenommen und festgestellt, daß sich die Austernfischerei auf Grund der Verödung der Austernbänke nicht mehr lohne. In einem Schreiben an den Landwirtschaftsminister berichtete Wilhelm Mielck im Frühjahr 1924 über zurückliegende Versuche Hagmeiers, Saataustern in einem kleinen Zuchtbecken und in den Ruinen des U-Boot-Hafens zu ziehen (39). Die Mittel waren damals zu gering, um Apparate zur Wasserbewegung und -erneuerung zu beschaffen. Mielck stellte wirtschaftliche Erfolge für Deutschland in Aussicht, wenn es der Anstalt gelänge, Saataustern auf Helgoland oder. in List auf Sylt zu züchten, denn man rechne mit sehr hohen Einnahmen für die Staatskasse. Hintergrund der Versprechungen war die Bitte um Genehmigung besonderer Anzuchtbecken. 
Im Frühjahr 1924 konnte in List auf Sylt in den Räumen der fiskalischen Austernanlagen ein Zweiglaboratorium der Anstalt untergebracht werden. Hagmeier formulierte folgende Aufgaben:

1. wissenschaftliche und fischereiliche Untersuchungen über den Zustand der fiskalischen Austernbänke;

2. Ausarbeitung von praktisch brauchbaren Methoden für die künstliche Austernzucht.

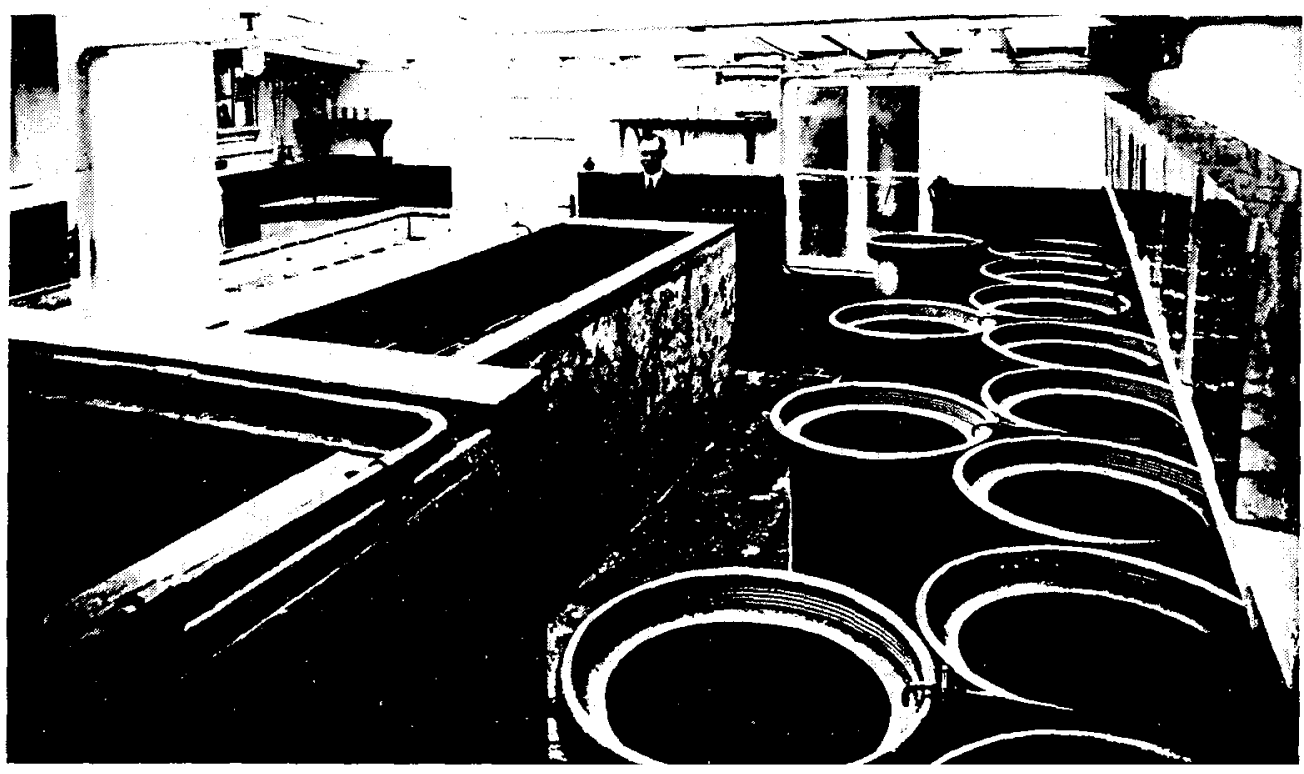

Abb. 37. Innenansicht des Aquariumshauses auf Sylt mit Becken für die Austernanzucht. Aufnahme um 1934. Stiftung Preußischer Kulturbesitz.

Hagmeier und Kändler dehnten ihre Arbeiten zur Bestandsaufnahme der Bodentierwelt der südlichen Nordsee und der Ostsee auch auf das Wattenmeer aus und untersuchten die nordfriesischen Austernbänke besonders intensiv. Dieses Gebiet wurde gewählt, da hier die Prüfung der chemisch-physikalischen Bedingungen und ihre Einflüsse auf Artenbild und Bestandsdichte der Bodentiere etwas einfacher ist als im offenen Meer. Hagmeier berichtete in mehreren Veröffentlichungen über die Arbeiten mit dem Austerndampfer „Gelbstern" und der "Augusta" im nordfriesischen Wattenmeer nördlich der Halbinsel Eiderstedt. Die „Gelbstern“" war mit sechs Austernkratzern ausgerüstet, die über das breite Heck des Dampfers ausgesetzt und eingeholt wurden. Muschelschalen, Schwämme und andere Tiere wurden entfernt, die Austern ausgesucht. Später wurden mit Petersen-Bodengreifern an fünf Stationen Proben entnommen. Hagmeier untersuchte zusammen mit R. Kändler die Proben quantitativ und zog aus dem Ergebnis Schlußfolgerungen über die Zusammensetzung von Lebensgemeinschaften. Auf diese Weise wurden die klassischen Untersuchungen an Austernbänken, die Möbius 1885 begonnen hatte, fortgeführt. Sie dienten aber nicht nur dazu, ökologische Kenntnisse zu erlangen, sondern auch der Erforschung von Einzelorganismen. Außerdem gab es noch einen sehr praktischen Hintergrund, so wollte man durch Austernzüchtung die geringe Ergiebigkeit einstiger Bänke ausgleichen und Importe einsparen. Die Wissenschaftler konstatierten: 
„So hat sich gezeigt, daß nicht nur die durch Möbius bekannte Lebensgemeinschaft der auf dem Meeresboden liegenden Tiere für die Austernbänke eine besondere ist, sondern daß in unserem Wattenmeer auch die kleinen, im Boden einer Austernbank lebenden Tiere zu einer besonderen Tiergemeinschaft, einer Untergruppe der Tellina-baltica-Gemeinschaft, gerechnet werden müssen" (Hagmeier und Kändler 1926).

Solange diese Lebensgemeinschaft vorhanden sei, so schlußfolgerten die Verfasser, könnten Austern gedeihen. Kändler bemühte sich, eine zuverlässige Methode zur Aufzucht planktonisch lebender Larven ausfindig zu machen, um die Erfahrungen in der Austernanzucht anzuwenden.

Parallel zu den zoologischen Arbeiten wurden in der Anstalt botanische Forschungen begonnen. Bereits mit der Stationsgründung bemühte sich Paul Kuckuck, in Helgoland die botanische Meeresforschung aufzubauen. Hierbei konnte er an Arbeiten Nathanael Pringsheims anknüpfen, der seit 1852 auf Helgoland algologische Studien betrieben hatte. Zwar hatte Pringsheim seine grundlegenden Entdeckungen über die geschlechtliche Fortpflanzung der Algen an Süßwasserformen gemacht, aber auch bedeutsame Arbeiten zur Morphologie der Meeresalgen veröffentlicht.

Paul Kuckuck konzentrierte seine Arbeit auf Untersuchungen über die Phanerogamenflora der deutschen Nordseeküste und die Algenflora der Nordsee, untersuchte aber auch andere Meeresabschnitte. Auf mehreren großen Expeditionen und durch Tausch gelang es ihm, Material für seine große Arbeit über „Phaeosporeen“ (Braunalgen) zu bekommen. Sie blieb durch seinen frühen Tod im Jahre 1918 unvollendet und wurde zehn Jahre später von Nienburg als Fragment herausgegeben.

Kuckuck begann frühzeitig damit, wissenschaftliche Herbarien anzulegen, so unternahm er mehrere Exkursionen zu den Ostfriesischen Inseln, um ein Inselherbarium zusammenzustellen. Über die Einrichtung wissenschaftlicher Herbarien hinaus bemühte er sich, Schausammlungen zu gestalten, um Besuchern des Museums auch die Flora der Nordsee näherzubringen. Im Jahre 1910 entwickelte er zusammen mit Heincke den Plan, drei Herbarien zu schaffen, die auch der Öffentlichkeit vorgestellt werden sollten: 1 . ein Helgoländer Lokalherbarium, 2. ein deutsches Herbarium, das Algen der deutschen Meere umfassen sollte, und 3. ein allgemeines Herbarium aus Algen aller Meere.

Es spricht für die Wertschätzung, die der Arbeit Kuckucks entgegengebracht wurde, daß das allgemeine Algenherbar um Schenkungen von Wissenschaftlern aus aller Welt erweitert wurde. So spendeten Kollegen südafrikanische Meeresalgen, Algen von der schwedischen Westküste, japanische und englische Arten, Arten der Braunalge Fucus von der französischen Küste. Außerdem schickten Kollegen wissenschaftliche Literatur, wie die Sammlung „Phycotheca boreali-americana“ und die „Wiener Algendekaden“.

Paul Kuckuck beschäftigte sich jedoch nicht nur mit Algen, sondern widmete sich auch höheren Pflanzen. So unternahm er große Anstrengungen, Pflanzen wärmerer Zonen auf Helgoland einzugewöhnen. Grundlage für seine Experimente, über die er in einer 1910 veröffentlichten Arbeit ausführlich berichtete, waren das Klima der Insel und die günstigen Bodenverhältnisse. Einige Erfahrungen lagen bereits vor, so hatte der Anbau von Feigen eine lange Tradition auf Helgoland. Berühmt war das Spalier des Konsul John Bufe. Der schönste Feigenbaum der Insel stand auf dem Oberlande im Gartenhof der Siemenschen Molkerei, der Stamm hatte einen Umfang von $94 \mathrm{~cm}$ (vgl. Mollenhauer und Lüning 1988). 1901 begann er Versuche mit ostasiatischen Gewächsen, besonders von den Küsten Japans, aber auch Chinas, später testete er Vertreter der chilenischen Regenküste und mediterrane Arten. 1904 richtete er mit Hilfe von Kollegen aus dem In- und Ausland einen kleinen Versuchsgarten ein, Experten aus Dahlem, Dresden, La Mortola, Antibes und Straßburg halfen ihm mit Ratschlägen, Auskünften, aber auch kostenlosen Pflanzensendungen. 
Kuckuck konnte einige höhere Pflanzenarten auf Helgoland heimisch machen, so entwickelten sich verschiedene Coniferenarten wie Pinus insignis und Cypressus macrocarpa gut, des weiteren Rosen, die schönen Sträucher von Evonymus japonicus und strauchartige Veronica-Arten. Mit seiner Anlage von Strandpflanzen wollte Kuckuck auch anderen Botanikern Anregung geben, die im Sommer die Insel besuchten. Außerdem hatten seine Bemühungen noch einen praktischen Grund - er wollte mit der Anpflanzung von Strandweizen, der Überflutungen besser verträgt als Strandhafer, die gefährdete Düne schützen und somit die Bemühungen der Königlichen Wasserbauinspektion in Tönning unterstützen.

Schon zu Lebzeiten Kuckucks war der von ihm unter großem finanziellen Aufwand angelegte botanische Garten durch Pläne, darauf ein großes Logierhaus für Badegäste zu errichten, gefährdet. Der botanische Garten wurde an einem anderem Platz neu angelegt, jedoch im Kriege wieder verwüstet. Der Wissenschaftler gab nicht auf und legte den Garten 1918 abermals neu an. Im selben Jahr verstarb Kuckuck.

Nach dem Tode Kuckucks war es zunächst nicht möglich, erneut einen Botaniker für Helgoland zu gewinnen. Erst 1921 konnte die Stelle mit Wilhelm Nienburg besetzt werden, der allerdings schon zwei Jahre später aus gesundheitlichen Gründen nach Kiel überwechselte. Nienburg befaßte sich u. a. mit der Polarisation keimender Fucus - Eier durch Licht und arbeitete über die Entwicklung einer anderen Helgoländer Braunalge, Haplospora.

In den zwanziger Jahren wurde ökologischen Aspekten mehr und mehr Beachtung geschenkt. Hierfür war vermutlich neben dem wissenschaftlichen Faktor, den Organismus in Beziehung zu anderen Gliedern der Lebensgemeinschaft im Sinne einer ganzheitlichen Betrachtung zu sehen, ein in Deutschland steigendes Interesse an Ökologie entscheidend. Warum dies erst etwa 40 Jahre nach den fundamentalen Arbeiten von Möbius (vgl. Möbius 1877) und Junge (vgl. Junge 1885) geschah, hat sicher mit der allgemeinen kulturellen Situation zu tun, die von Reformbestrebungen und einer Natursehnsucht gekennzeichnet war.

Schreiber arbeitete ab 1925 an der Anstalt. Zwischen 1927 und 1929 versuchte er, sogenannte „Produktionspotentiale" von untersuchten Wassergebieten abzuschätzen, was von höchstem Interesse für die Suche nach neuen Nahrungsquellen im Meere war. Nach einer von ihm 1927-29 entwickelten Methode wurden sterilisierte Seewasserproben mit bakterienfreien Kulturen von Planktonalgen beimpft, aus deren Wachstum dann der Gehalt des Wassers an physiologisch verwertbaren Stickstoff- und Phosphorverbindungen genauer ermittelt werden konnten, als es mit den damals zur Verfügung stehenden chemischen Methoden möglich war. Aus diesen beiden Faktoren, dem Phosphor- und Stickstoffgehalt, schloß man auf das Produktionspotential der untersuchten Gebiete (vgl. Schreiber 1927).

Schreiber beschäftigte sich vorrangig mit der Entwicklungsgeschichte der Algen und führte gleichzeitig Untersuchungen zur Ernährungsphysiologie durch. Er führte eine bis heute weltweit verwendete Nährlösung für die Algenzucht ein, die "Schreiberlösung". Bei den Braunalgen entdeckte er den Entwicklungszyklus von Desmarestia und wies die genotypische Geschlechtsbestimmung von Dictoyota und Laminaria im Kulturexperiment nach. Auch Bestrebungen Kuckucks, Algen in Freikulturen zu züchten, wurden weiterverfolgt. Die Methode der Algenzüchtung der verschiedenen Generationen und Entwicklungsstadien der Algen lieferte ein wertvolles Hilfsmittel für andere Gebiete der Biologie. In einer 1925 erschienenen Arbeit war die Algenbesiedlung erstmalig in Abhängigkeit von den ökologischen Faktoren dargestellt worden. Eine wichtige Ergänzung dieser Schilderung stellten die 1928 veröffentlichten Vegetationsbilder dar. Leider ging das Manuskript während des II. Weltkrieges verloren. 
Schreiber verließ Anfang der dreißiger Jahre wegen einer rheumatischen Wirbelsäulenerkrankung die Insel, sein Arbeitsgebiet wurde von Peter Kornmann weitergeführt, der 1938 entdeckte, daß die Grünalgen Derbesia marina und Halicystis ovalis Glieder eines Lebenszyklus sind (vgl. Kornmann 1938). Der entwicklungsgeschichtliche Zusammenhang der morphologisch so unterschiedlichen Grünalgen war eine aufsehenerregende Entdeckung.

Mit dem Beginn des II. Weltkrieges wurden die botanischen Arbeiten unterbrochen und konnten erst ab 1950 von Kornmann fortgeführt werden.

Belegt ist, daß Mitarbeiter der Anstalt an der Gewinnung von Meeresalgen und Seegras als Futtermittel beteiligt waren. Während des I. Weltkrieges wurden große Anstrengungen unternommen, Ersatz für Lebens- und Futtermittel zu finden, so wurde bereits im August 1915 ein sogenannter Kriegsausschuß für Ersatzfutter G.m.b.H. gegründet. Die Abteilung V des Ausschusses befaßte sich mit der Futtermittelgewinnung aus Seegras und Seetang. Der Bundesrat beschloß eine „Verordnung über Seegras und Seetang“, auf deren Grundlage dieses Material nicht nur vor den deutschen Küsten beschlagnahmt, sondern auch aus dem neutralen Auslande eingeführt wurde (141). Wie Wilhelm Mielck nach dem I. Weltkrieg eindrucksvoll schilderte, bemühten sich die Mitarbeiter mit Unterstützung der Marine, größere Mengen des vom Sturm an Land geworfenen Materials zu gewinnen. Die Tange wurden von Marinemannschaften zum Trocknen in der Sonne ausgebreitet, in Pressen zu Ballen geformt und dann in Mühlen auf dem Festland verarbeitet. Daß der Ertrag geringer war, als man erhofft hatte, führte Mielck - wie wir heute wissen, fälschlicherweise - auf Schwierigkeiten bei der Lufttrocknung zurück. Insgesamt wurden die Versuche vom Ausschuß jedoch nach anfänglichen Schwierigkeiten als erfolgreich bezeichnet, so wurden z. B. 1918 ca. $250000 \mathrm{~kg}$ Seetangmehl gewonnen. Allerdings erwiesen sich die Algen als ungeeignetes Ersatzfutter für Unpaarhufer und Wiederkäuer, da sie wegen des starken fischähnlichen Beigeschmacks verweigert wurden.

\subsection{Aufgaben im Dienste der internationalen Meeresforschung}

\subsubsection{Das Forschungsprogramm von Kristiania}

Aus den Forschungsergebnissen der Anstalt ergaben sich frühzeitig praktische Empfehlungen für die Fischerei. Schon ab 1892 sprachen sich sowohl Heincke als auch Ehrenbaum in ihren wissenschaftlichen Arbeiten, aber auch auf Konferenzen dafür aus, Bestände schonend zu befischen.

Ein wesentlicher Verdienst Ehrenbaums war die Einführung eines Minimalmaßes für marktfähigen Hummer, Homarus gammarus ( $9 \mathrm{~cm}$ Länge des Kopf-Brustschildes). Dieses Maß wurde durch Verfügung der Regierung von Schleswig am 10.3. 1894 festgelegt. Ehrenbaum empfahl außerdem, eiertragende Weibchen so lange zu hältern, bis die Larven geschlüpft waren. Diese Regeln werden auch heute noch beachtet, das schon 1922 per Verordnung festgeschriebene Hummerschutzgebiet entspricht etwa dem heutigen Naturschutzgebiet.

Weitere Bemühungen der Biologen galten den Austernbeständen. Die Austernbänke, die vor dem I. Weltkrieg noch als sehr ertragreich beschrieben wurden, verödeten nach Berichten Erdmanns zunehmend, so wurden für Hunderttausende Mark Austern aus dem Ausland eingeführt. Seit 1912 wurden Versuche angestellt, die Austernbänke durch künstliche Anzucht von Jungaustern wieder zu bevölkern. Diese Arbeiten wurden nach dem I. Weltkrieg u. a. von Hagmeier, Kändler und Erdmann fortgesetzt. 
Sorge bereitete die Verschlechterung der Fischfänge, die auf Lichtung der Bestände durch „Überfischung" zurückgeführt wurde. Insbesondere nach den Arbeiten Heinckes über die Wanderungen des Herings und seine Hypothese der Lokalrassen waren Theorien über sehr weite Wanderungen der Nutzfische widerlegt. Arbeiten ausländischer Forscher, vor allem schwedischer und norwegischer, wiesen auf einen Zusammenhang zwischen Wanderung und Faktoren wie Salzgehalt, Strömung usw. hin.

Die große öffentliche Diskussion um den Zustand der Seefischerei und Überlegungen, der internationalen Meereskommission beizutreten, veranlaßte das Innenministerium, den Deutschen Seefischerei-Verein um ein Gutachten zu bitten. Dieses Schriftstück wurde, obwohl es gedruckt wurde, dann doch nicht verbreitet - im Interesse der deutschen Wissenschaft, wie es hieß. In diesem Gutachten wurde die Forderung nach einem gemeinsamen Bestandsschutz aller Nord- und Ostseeanliegerstaaten nach einem abgestimmten Plan geäußert. Man schlug vor, das schwedische Programm hinsichtlich der Überfischungsfrage zu erweitern. Es wurde auf die Ergebnisse Heinckes und anderer Wissenschaftler verwiesen und festgestellt, daß nach neuen Ergebnissen die alte These von der Unerschöpflichkeit der Meere nicht stimme und man einen einheitlichen Plan zum Bestandsschutz für richtig halte. Zur Begründung hieß es:

„Eine solche Forderung rechtfertigt sich nicht nur aus Gründen der allgemeinen Wissenschaft, sondern im besonderen aus der immer steigenden, nationalökonomischen Bedeutung der Seefischerei und der in immer drohendere Nähe gerückten Möglichkeit einer Überfischung der nordischen Meere und der Nothwendigkeit einer internationalen Fischereigesetzgebung" (anonym, o. D.).

Bereits vor 1900 wurden u. a. von Heincke Entwürfe darüber geäußert, wie man Arbeiten zur Meeresforschung koordinieren könne. Zusammen mit den Professoren Hensen, Herwig und dem Geographen und Meereskundler Otto Krümmel ${ }^{143}$ nahm Heincke an der ersten internationalen Konferenz zur Erforschung des Meeres teil. Der Präsident der Konferenz umriß in seiner Eröffnungsrede das Anliegen wie folgt:

„Erst nach jahrelangen isolierten Bestrebungen ist die Erkenntnis gereift, daß es eines Zusammenwirkens bedarf der einzelnen Länder, daß jedes seinen Antheil übernimmt der erforderlichen gemeinsamen Arbeit an der Aufgabe, nämlich die Erforschung des Meeres, des vielgestaltigen Lebens, das sich da unten regt, und der Verhältnisse, die sein Dasein bedingen" (Steen 1901).

Über das ökonomische Ziel der Abstimmung wurde kein Zweifel gelassen, es galt, das Meer als Erwerbsquelle besser und ökonomischer zu nutzen:

„Das Ziel ist dieses, den Leuten, die das Meer befahren, zu verhelfen, das Feld ihrer Thätigkeit kennenzulernen und auszuwählen, wie der Bauer seinen Acker und seine Wiese" (ebenda).

Eine Aufgabe der Meeresforschung wurde darin gesehen, Regelungen und Absprachen zwischen allen Anliegerstaaten beim Übergang von der Küsten- zur Hochseefischerei zu treffen. Es galt auch, wie Hagmeier später einschätzte, Deutschland den ihm zustehenden Anteil an dem Ertrag der internationalen Hochsee zu sichern (vgl. Hagmeier 1942).

Dadurch trat eine Verknüpfung der Forschung mit politischen Auseinandersetzungen, die mit wirtschaftlichen Mitteln ausgetragen wurden, in den Vordergrund. Das kam darin zum Ausdruck, daß die Arbeiten auf dem Gebiet der angewandten Fischereiforschung vom Landwirtschaftsministerium bezahlt wurden. Die auf Deutschland entfallenden Arbeiten wurden innerhalb der „Deutschen wissenschaftlichen Kommission für Meeresforschung“ organisiert. Diese Kommission war wesentlich durch das Engagement Heinckes zustande

143 Otto Krümmel (1854-1912) 
gekommen (125). Grundlage war das in Kristiania verabschiedete Programm, in dem sich Großbritannien, Irland, Deutschland, Rußland, Norwegen, Schweden, Dänemark, die Niederlande und Belgien mit dem Ziel der Erforschung der nordeuropäischen Meere im Dienste der Seefischerei zusammenschlossen. Das Programm sah die Erforschung des Meeres im Sinne des heutigen Begriffs der Grundlagenforschung und im Dienste der Seefischerei vor.

Heincke zog zur Begründung rein wissenschaftliche Aspekte heran. So die Tatsache, daß die Erforschung des Meeres eine internationale Aufgabe sei und durch ein einzelnes Land nicht getragen werden könne. Untersuchungen müßten in einem sehr großen Gebiet unternommen werden.

An der Präzisierung des biologischen Teils des in Kristiania beschlossenen Arbeitsprogrammes hatten Heincke, Herwig, Brandt und Krümmel großen Anteil. In einem 1901 gedruckten „Antrag, betreffend das Programm für den biologischen Theil der internationalen Meeresuntersuchungen" stellten sie ihr Programm vor (91). Danach wurden die Arbeitsgebiete so aufgeteilt, daß Deutschland zusammen mit Dänemark ein Teil der Nordsee zwischen 54 Grad und 58 Grad nördlicher Breite zufiel sowie ein Teil der Nordsee südlich vom 54 Grad nördlicher Breite (vom Borkumriff bis zur Elbe). Ein Teil der westlichen Ostsee sollte zusammen mit Schweden und Dänemark untersucht werden. Es wurde jedoch darauf verwiesen, daß eine scharfe Abgrenzung der den einzelnen Staaten zugewiesenen Gebiete nicht möglich sei. Es war geplant, daß jeder Staat folgende Untersuchungen auf seinem Gebiet ausführen sollte:

1. Qualitative und quantitative Plankton-Studien nebst Untersuchungen über die im Wasser gelösten Nährstoffe.

2. Untersuchung der chemischen, physikalischen, zoologisch-botanischen und bakteriologischen Beschaffenheit des Meeresgrundes und Untersuchung über Art und Grad der Besiedelung des Bodens.

3. Qualitative und quantitative Bestimmung der treibenden Eier und Larven der Fische, insbesondere von Nutzfischen. An der Präzisierung dieses Punktes war Heincke maßgeblich beteiligt. Im Labor sollten Versuche zur künstlichen Befruchtung von Nutzfischeiern durchgeführt werden. Die im Meer abgelegten Eier und die daraus schlüpfenden Arten der Nutzfische sollten hinsichtlich ihres Vorkommens registriert werden. Die quantitativen Fänge von treibenden Eiern und Larven wollte man mit Vertikalnetzen durchführen, die auf den Entwurf von Professor Hensen zurückgingen.

4. Es war an eine planmäßige Untersuchung der wichtigsten Nutzfische vom Ei bis zur reifen, fortpflanzungsfähigen Form gedacht. Entwicklung, Wachstum, Ernährung, Fortpflanzung, Verbreitung, Wanderungen, Lokalformen sollten untersucht werden. Hierbei vereinbarte man die Untersuchung ausgewählter Nutzfische, so wurden für die Nordsee Scholle (Pleuronectes) und Schellfisch (Melanogrammus) vorgeschlagen, für die Ostsee Dorsch (Gadus), Scholle und Flunder (Platichthys), für den nördlichen Teil des Gebietes wählte man den Kabeljau (großer Dorsch, Gadus) aus. Untersuchungen über den Hering (Clupea) sollten von allen Beteiligten durchgeführt werden.

5. Es sollte mittels Versuchsfischerei verschiedenster Art die Besiedlung von Meeresteilen durch die genannten Nutzfische erforscht werden. Dabei sollten Ort, Tiefe, Beschaffenheit des Fanggrundes und des Wassers, Wind, Wetter, Schnelligkeit der Fahrt und Dauer des Fanges, Art und Größe des Schleppnetzes genau notiert werden. Ebenso galt es, eine genaue Analyse der Fänge nach Fischarten, Zahl, Größe 
und Reife der Fische durchzuführen. Es kam darauf an, möglichst übereinstimmende Fanggeräte zu wählen und identische Schemata für die Fangjournale zu verwenden.

Hier war daran gedacht, Lokalformen (Abarten, Rassen) der Scholle zu bestimmen und die Wanderungen derselben zu verfolgen. Außerdem sollte herausgefunden werden, ob es im Untersuchungsgebiet nur eine einzige Schollenrasse gibt oder mehrere, getrennte Bezirke bewohnende Rassen. Wenn es solche Wanderungen gab, wollte man prüfen, wie weit die Wanderungen jeder dieser Gruppen in ihrem Bezirk gehen. Man wollte an möglichst vielen Orten speziell markierte Schollen aussetzen, um für jede einzelne Lokalform Laichplätze zu ermitteln. Treibende Eier und Larven der Scholle sollten im ganzen Untersuchungsgebiet festgestellt werden. Auch für die Metamorphose und das erste Lebensjahr (wenn die symmetrische Larve in die Metamorphose zur asymmetrischen Form eintritt, geht sie von planktonischer Lebensweise zum Aufenthalt am Boden über) interessierte man sich, des weiteren für den Aufenthalt und Wanderungen der jungen, unreifen, $d . h$. untermaßigen Schollen. Durch das Aussetzen gezeichneter Schollen hoffte man, Erkenntnisse über die Lebensweise zu gewinnen. So darüber, wann die Tiere geschlechtsreif werden, wie groß die Keimfruchtbarkeit ist usw. Ähnliche Programme wurden für den Hering entworfen.

Die Erstellung einer kritischen Fangstatistik sowie die Ermittlung der Größe der Fänge untermaßiger Nutzfische war ein weiterer Programmpunkt.

$\mathrm{Zu}$ Plankton- und Bodenuntersuchungen erklärte man folgende Forschungen vornehmen zu wollen:

a) qualitative Untersuchung zur horizontalen und vertikalen Verbreitung einzelner Arten von Plankton-Organismen, zur Abhängigkeit der verschiedenen Organismen von biologischen Faktoren wie der Einfluß von Wasserbewegung, Licht, Temperatur, Salzgehalt usw.

b) quantitative Plankton-Untersuchungen zur Bestimmung des Verhältnisses des Nahrungsproduzenten zum Konsumenten, wobei auch chemische Untersuchungen zur Bestimmung von Trockengewicht, Fettanteil, Asche usw. durchgeführt werden sollten sowie die chemische Zusammensetzung von einzelnen wichtigen PlanktonKomponenten wie den Kieselalgen (Diatomea) bestimmt werden sollten.

Des weiteren wollte man folgende Gesichtspunkte berücksichtigen: Ermittlung von Schnelligkeit und Stärke der Fortpflanzung und der Wachstumsgeschwindigkeit usw. für die wichtigsten Planktonorganismen, andererseits den Verbrauch des Planktons durch Fische und andere Nutztiere.

Die Bodenuntersuchungen sollten durch Entnahme von Grundproben durchgeführt werden. Es wurde angestrebt, Analysemethoden aus allen Fachrichtungen miteinander zu vereinigen, um den Lebensraum möglichst in physikalisch-chemischer, aber auch zoologisch-botanischer Richtung komplex zu erfassen (91).

Dieses Programm bildete den Rahmen für Untersuchungen an der Anstalt. Heincke bemerkte in seinem vor dem Kriege gegebenen Forschungsbericht:

"Wie in den Vorjahren standen die Forschungsaufgaben auch in den vier Jahren 1910 bis 1914 wesentlich unter dem Einfluß der Teilnahme der Anstalt an der internationalen Meeresforschung, an der sich alle mit Ausnahme des Botanikers mehr oder weniger beteiligten" (22).

Schwerpunkte waren die Forschungen zur Lebenskunde einzelner Nutztiere und die Untersuchung der Wechselwirkung zwischen Fischbestand und dem Ertrag der Fischerei. Heincke hatte bereits vor Gründung der Station ausführliche Untersuchungen über die 
Naturgeschichte des Herings und der Sprotte angestellt. Er führte diese Arbeiten weiter und bezog alle Mitarbeiter ein, vor allem Ernst Ehrenbaum.

Im Mittelpunkt der Untersuchungen stand die Erforschung der Fischgründe der Nordsee. Das Interesse galt zunächst dem Hering, hier führte Heincke seine vorher begonnenen Arbeiten fort. Noch mehr als fünfzig Jahre später schätzte Hagmeier ein, daß Heincke durch seine Diagnosen der Lokalformen des Herings durch Anwendung der Variationsstatistik bei der Untersuchung der Rassenmerkmale zu der wichtigen Erkenntnis gelangte, daß Heringe nicht aus dem Ozean kommen, sondern verschiedene Lokalrassen bilden, die an körperlichen Merkmalen erkannt und bei ihren Wanderungen verfolgt werden können.

Aber auch über Hummer und Garnelen, später Aal (Anguilla), Seezunge (Solea), Heilbutt (Hippoglossus) und vor allem über die Scholle wurde gearbeitet. In den Jahren bis zum I. Weltkrieg konnten zahlreiche Arbeiten zur Entwicklung wichtiger Nutzfische veröffentlicht werden, so über Eier und Jugendformen von Seezunge und Flunder. Mit der Einrichtung eines Aquariums in der Anstalt im Jahre 1902 wurde es möglich, so berichtete Heincke 1906, von den meisten Nutzfischarten der Nordsee eine lückenlose Reihe von Jungfischen bis zum Ende des Larvenstadiums zu erhalten. Man untersuchte die Verbreitung der schwimmenden Fischeier in der Nord- und Ostsee (Arbeiten von Mielck, Ehrenbaum und Strodtmann) und bestimmte die Menge der freischwebenden, sich nach physikalischen Gesetzen gleichmäßig im Wasser verteilenden Fischeier mit einer Methode, die Hensen in die Planktonforschung eingeführt hatte (vgl. Hagmeier 1942).

Von besonderem Interesse waren natürlich Erkenntnisse über Lebensverhältnisse von Fischen, so Laichzeit, Laichplätze, Wanderungen, Verteilung der Altersstufen und Größen, Verbreitung und Nahrung.

Hier galt etwa ab 1907 das besondere Interesse den sogenannten wichtigen „Wirtschaftsfischen" Scholle und Flunder. Im Jahre 1908 veröffentlichten Heincke und Hermann Henking ${ }^{144}$, der sich um die Entwicklung der Seefischerei als selbständigen Wissenschaftszweig verdient machte (vgl. P. F. Meyer 1942), zusammen die Schrift „Schollen und Schollenfischerei in der südöstlichen Nordsee“. Diese Studie stieß auf großes Interesse, steckte die Fischereiwirtschaft doch in wirtschaftlichen Schwierigkeiten. Wie aus einem Schreiben an den Reichskanzler 1909 hervorgeht, waren 1908 die Fänge hinter den Erwartungen zurückgeblieben, und man hatte sogar Heringe aus dem Auslande einführen müssen (138).

Wichtige wissenschaftliche Methoden zum Studium der Lebensweise war die Verfolgung des Fischzuges mittels markierter Tiere, Bestimmungen von Längen-Dicken-Koeffizienten und sogenannte Transplantationsversuche.

Über die Arbeiten, insbesondere die Scholle betreffend, wurde in den Berichten der Deutschen Wissenschaftlichen Kommission für Meeresforschung ausführlich Rechenschaft abgelegt. Heincke teilte mit, daß die Biologie dieses wichtigen Nutzfisches der Nordsee bearbeitet wurde, so habe man sich sowohl mit der Bildung und Reifung der Eier, ihrer Zahl und Verbreitung befaßt als auch mit der Verbreitung der verschiedenen Altersstadien markierter Schollen.

Diese fischereizoologischen Arbeiten wurden bis zum I. Weltkrieg fortgeführt. Heincke organisierte mit seinen Assistenten Keilhack und Thielemann Untersuchungen zur Altersbestimmung und Naturgeschichte der Scholle, wobei Schollenschwärme in der Nordsee nach Größen und Altersstufen, das Wachstum und die Wanderungen der Schollen sowie die verschiedenen Lokalformen in der Nordsee und den angrenzenden Meeren untersucht wurden. Diese Ergebnisse hatten, so hob Heincke in seinem Bericht hervor, große Bedeutung

144 Hermann Henking (1858-1942) 
für den angestrebten Bestandsschutz. Besonders die Überfischungsfrage und sich daraus ergebende Teilfragen wie die Wirkung der Befischung auf den Bestand, auf die Menge der untermaßig vernichteten Fische, die Wirkung von Schonmaßnahmen und anderes wurde diskutiert. Die Resultate gingen in den von Heincke 1913 vorgelegten Generalbericht zur Scholle ein. Diesen Bericht legte Heincke als Generalreferent des Zentralausschusses für Schollen vor.

Direkt mit der zoologischen Forschung war die Planktonforschung verbunden. Sie hatte in Deutschland eine u. a. durch Haeckel mitbegründete Tradition, die von Hensen in anderer Weise fortgeführt wurde. Es ging einerseits um die qualitative Untersuchung des Planktons, andererseits um die quantitative. Hensen hatte die gedanklichen und methodischen Grundlagen geschaffen, folgenden Zielen zu folgen:

- die Feststellung der Laichzeiten und -plätze der verschiedenen Fischarten sowie

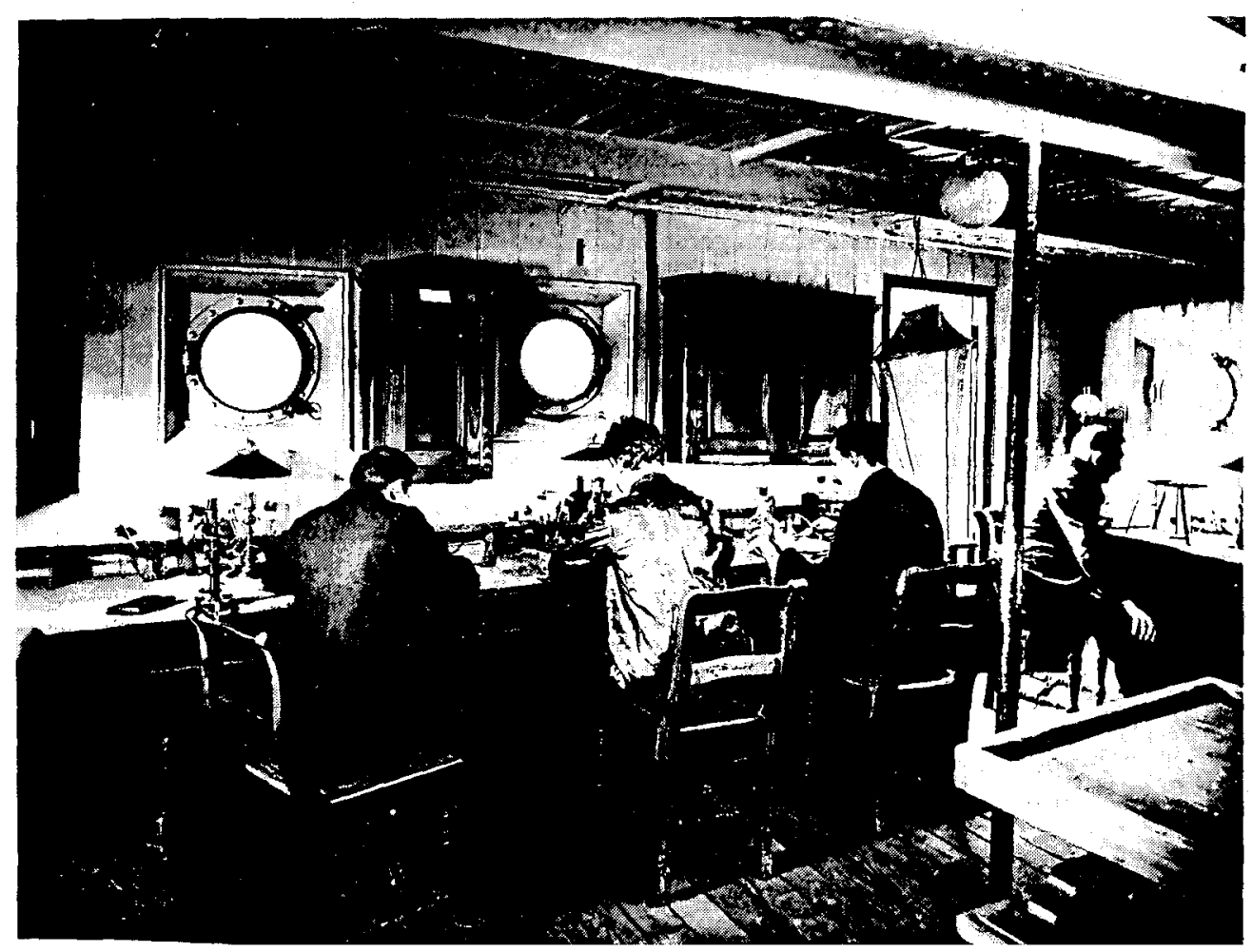

ADD. उr. wissenschaftliche Untersuchungen an Bord des Reichsforschungsdampfers "Poseidon”. (Anfang des 20. Jahrhunderts). Archiv der Biologischen Anstalt Helgoland.

ihrer Laichbedingungen auf Grund der Phänologie und Verbreitung ihrer pelagischen Entwicklungsstadien,

- die Bestimmung der Fischbestände und ihrer Schwankungen aus der Verbreitungsdichte der planktischen Eier. 
Aus der Erkenntnis, daß Bestandschwankungen erwachsener Tiere in starkem Maße durch die pelagische Entwicklungsphase bedingt sind, erwuchsen neue Fragestellungen. Die Lebenserwartung der jüngsten Stadien ist in besonderem Maße von physikalischen und biologischen Umweltverhältnissen abhängig. Einzelne Meeresgebiete wurden unter Berücksichtigung ihrer Planktongemeinschaften aufgegliedert, es stellte sich heraus, da 3 aus der Verbreitung bestimmter, gegenüber Milieuveränderungen widerstandsfähiger Arten, den sogenannten „Leitformen“, Rückschlüsse auf die Herkunft bestimmter Wasserkörper möglich waren.

Ernst Ehrenbaum sah den Wert der Planktonforschung darin, daß

„Entwicklungs- und Larvenformen verschiedener Organismen reiches Material, für das Studium liefere. Außerdem ist es von besonderer Bedeutung, das Regelmäßige und periodisch Wechselnde in seiner Zusammensetzung kennenzulernen. Darin liegt der Schlüssel für viele andere rätselhafte biologische Erscheinungen und die genaue Kenntnis dieser sogenannten Urnahrung des Meeres bildet ein wichtiges Glied für das Verständnis vom Leben im Meer und der Mannigfaltigkeit seiner Formen“ (Ehrenbaum 1908).

Im Jahre 1845 hatte Johannes Müller als erster mit einem feinmaschigen Netz nach den kleinsten schwebenden Organismen gefischt und mit seinen Untersuchungen der ganz anders als erwachsene Seeigel und Seesterne aussehenden Echinodermen-Larven begonnen. Hensen regte unter dem Gesichtspunkt der Ertragsabschätzung des Meeres eine zielbewußte Planktonforschung an. Er hatte in seinen 1890 veröffentlichten ersten Ergebnissen der Plankton-Expedition deutlich gemacht, daß es auf Ursachenforschung ankäme und komplexe Untersuchungen, zu denen auch hydrographische Forschungen gehörten (Salzgehalt, Gasgehalt, Strömungen des Meerwassers usw.), angestellt werden müßten.

Hintergrund dieses Interesses war die Tatsache, daß Plankton als Futter für Fische begriffen wurde. Es ging darum, den „Reichtum unserer Nahrung“ in zureichender Annäherung festzustellen (vgl. Krümmel 1888), wobei die Verteilung der Nahrungsgrundlage Plankton wichtig war.

Bereits ab 1. April 1892 wurden von Mitarbeitern der Anstalt täglich Proben zur Untersuchung des Planktons im Oberflächenwasser durchgeführt. Genauere Untersuchungen nach Hensen konnten jedoch, so der Direktor in seinem Bericht von 1893, wegen Mangels an Arbeitskräften, die die Methodik beherrschten, nicht durchgeführt werden.

Mindestens seit 1907 führte die Anstalt in enger Zusammenarbeit mit der hydrographischen Abteilung des Instituts für Meereskunde in Berlin (Alfred Merz) gleichzeitige hydrographische und Planktonbestimmungen durch. Hier wurden Pläne von Adolf Reichard fortgeführt. An vier Terminen Anfang Mai, Anfang August, Anfang November und Anfang Februar 1910/11 wurden diese Untersuchungen auf Feuerschiffen „Amrum-Bank“, „Elbe I" und „Borkumriff" durchgeführt, jede dieser Untersuchungen dauerte 7 Tage und umfaßte Tag und Nacht in gleichen, ein- oder mehrstündigen Intervallen angestellte Tiefenserien, Beobachtungen der Temperatur, des Salzgehaltes, des Gasgehaltes und der Strömungen des Meerwassers. Die der biologischen Anstalt zufallende Bearbeitung der zugehörigen Planktonuntersuchungen sei im Gange, so Heincke in seinem Bericht, stoße aber noch immer auf Schwierigkeiten (8).

Es wurden drei Hauptaufgaben formuliert:

1. Eingehende Untersuchung über die Ausbildung bestimmter Planktongemeinschaften sowohl während des Jahresverlaufes wie auch in Wasserarten verschiedener Herkunft,

2. Systematik und jahreszeitlicher Wechsel der Formen des Nannoplanktons,

3. die Ernährung junger Fischlarven. 
Die Untersuchungen wurden von Mielck durchgeführt. Er verglich während seiner Fahrten in die Deutsche Bucht, die fast ausschließlich der Fischereibiologie galten, hydrographische Lage und Plankton. Stets ergab sich, daß vom Vorkommen bzw. Fehlen bestimmter Leitformen Rückschlüsse auf die Hydrographie bestimmter Gebiete gezogen werden konnten.

Außerdem machte er wesentliche Aussagen über das Vorkommen bestimmter Vertreter des Nannoplanktons im Darm von Fischlarven. Bereits während des I. Weltkrieges verwies Brandt auf die besondere Wichtigkeit der Planktonforschung, die auch volkswirtschaftlich Bedeutung hat, da sie durch eingehende vergleichende Untersuchungen Aufschlüsse über Reichtum und Armut an im Wasser schwebenden Organismen und damit über Gunst oder Ungunst der Produktionsbedingungen in den verschiedenen Gebieten und Jahreszeiten liefere.

Von Bedeutung sei auch die Untersuchung von Pflanzennährstoffen. Die Planktonforschung wurde ergänzt durch quantitative Untersuchungen der Bodenfauna. So veröffentlichte Hagmeier sogenannte „Bonitierungen des Meeresbodens“. Die Dynamik größerer Lebensgemeinschaften im Meer rückte in den Mittelpunkt, und Hagmeier stellte eine Liste von Faktoren zusammen, die den Bestand beeinflussen und die seiner Meinung nach weiter erforscht werden müßten. Er prägte in diesem Zusammenhang den Begriff von der „Kultur" anstelle der rücksichtslosen Ausbeutung der Meere.

Gleich bei Ausbruch des I. Weltkrieges wurde die Arbeit der Station unterbrochen. Ein Teil des wissenschaftlichen Personals trat in den Kriegsdienst, die übrigen Gelehrten und der Direktor siedelten um. In den folgenden fünf Jahren ruhte die wissenschaftliche Arbeit fast vollständig. Ungünstig wirkte sich aus, daß fast alle Wissenschaftler in verschiedenen Instituten auf dem Festland verteilt arbeiteten und ihnen sowohl die Bibliothek als auch Geräte nicht zur Verfügung standen. Mielck, der als Offizier auf Helgoland eingesetzt war, gelang es, dort die wissenschaftliche Arbeit in eingeschränktem Maße fortzuführen. Mit der Marine wurde vereinbart, $\mathrm{da} ß$ die wissenschaftlichen Untersuchungen mit der Beschaffung von Fischen für Nahrungszwecke (Versorgung der Marine, der Bevölkerung und der Angestellten der Anstalt) verknüpft wurde. Nach Kriegsende wurden auf Betreiben Heinckes die fischereibiologischen Arbeiten in verstärktem Maße weitergeführt. Untersuchungsobjekt war wiederum die Scholle. Wie Heincke bzw. Bückmann in ihren 1922 und 1925 veröffentlichten Arbeiten berichteten, hatte die Eizahl zugenommen, wie auch die Größe der Fische und ihr durchschnittliches Alter, das Wachstum aber war durch die Nahrungskonkurrenz im dichten Bestand verzögert. Die ursprüngliche Vermutung, daß diese Veränderung Folge der kriegsbedingten Schonzeit seien, stellte sich als falsch heraus. Mittels laufender Überwachung des Bestandes durch Stichproben fand man heraus, daß sich der Fischbestand nicht nur durch die Fischerei verändert, sondern auch dem Einfluß natürlich bedingter starker Schwankungen unterliegt. So entwickelt sich die alljährlich während der Laichzeit erzeugte Brut nicht jedesmal zu denselben Mengen an Jungfischen, sondern es gibt reiche, mittelstarke und arme Jahrgänge, was beträchtlichen Einfluß auf den Fischereiertrag hat. Hierzu wurden biologisch-statistische Untersuchungen an Fischmärkten durchgeführt, die Aufschluß über die Wirkung natürlicher Schwankungen in Nachwuchserzeugung und Wachstum auf den Fang von Schollen gaben. Auch örtliche Veränderungen der Fangplätze wurden erfaßt.

Es galt, die Ursache dieser Schwankungen herauszufinden. Der Fischbestand wurde im Sinne einer neuen ökologisch ausgerichteten Forschung als Organismus betrachtet. Man vermutete zu Recht, daß die Schwankungen schon bei den frühen Stadien der pelagischen Nutzfische einsetzten, und führte daher neue Untersuchungen über die pelagische Fischbrut, ihre Menge und Verteilung sowie über ihre Lebensbedingungen durch. Im Mit- 
telpunkt stand zunächst der Hering, später der Rotbarsch (Sebastes). Man erkannte die Notwendigkeit, die Fischbestände in ihrem natürlichen Lebensraum, den nordatlantischen Fischgründen, zu erforschen. Zur Deutung der komplexen Phänomene wurden nun alle Zweige der Meeresforschung herangezogen. Eine ganzheitliche Betrachtung wurde als nötig erkannt, es ging um die Erforschung des Lebensraumes Meer als ökologisches System und die Erforschung von Lebensgemeinschaften. Besonders wichtig waren hierbei Planktonforschung und die Hydrographie. Was die Hydrographie betrifft, so konnte während einer Fahrt mit dem Reichsforschungsdampfer „Poseidon“ im Jahre 1927 eine enge Beziehung zwischen hydrographischen Bedingungen und der Verteilung der Fische festgestellt werden.

Mit der „Poseidon“ wurden auch Untersuchungsfahrten nach der Barentsee und dem Weißen Meer durchgeführt. Die Barentsee, seit 1905 von England und Deutschland in geringem Maße befischt, interessierte Heincke, weil in den genannten Gebieten die Fische sehr alt wurden und sich interessante Ergebnisse durch Vergleich ergaben. Darüber hinaus wurden zahlreiche Fischerei-Versuche mit verschiedenen Netzen, aber auch Dredgezüge und Planktonfänge sowie ausgedehnte hydrographische Bestimmungen durchgeführt. Bereits 1913 wurden die Ostsee, die Nordsee und die Gewässer Islands als sehr stark befischt eingestuft. Im Vergleich dazu galt die Barentsee als „noch recht jungfräuliches“ Gebiet der Fischerei.

Damit gewann die Wahrnehmung internationaler Fischereiinteressen aus deutscher Sicht noch mehr an Bedeutung. Im Jahre 1913 plante und organisierte Heincke eine größere Untersuchungsfahrt in die Barentsee, deren Notwendigkeit er u. a. so begründete:

„Die wissenschaftliche Vergleichung eines solchen Fischbestandes mit den schon seit langer Zeit mehr oder weniger stark gelichteten Fischbeständen der Ostsee, der Nordsee und Islands mußte daher im höchsten Grade lehrreich sein und ließ wichtige und entscheidende Aufklärungen über brennende Fragen der Überfischung und der Schonmaßregeln für Nutzfische erwarten. Diese Erwägungen rechtfertigten die Unternehmung“ (Heincke 1917).

Heincke hatte festgestellt, daß die Schollen im Gebiet der Barentsee besonders alt wurden, was darauf hinwies, daß der Fischbestand in seiner Zusammensetzung aus Größenund Altersstufen ursprünglich geblieben und vom Menschen wenig beeinflußt worden war. Was den Zeitpunkt der groß angelegten Untersuchung betraf, so war nach Heinckes Meinung Eile geboten, da die Befischung der Barentsee die unberührten Fischbestände schon gelichtet hatte.

Es wurden insgesamt sieben Fahrten durchgeführt (u. a. bis zum englischen Kanal, nördliche und südliche Nordsee, Nordkap, Barentsee), deren wissenschaftliche Aufgaben, u. a. in Untersuchungen zur Verbreitung der Schollen mittels Markierungen und Jungfischfängen bestanden sowie in Untersuchungen von treibenden Fischeiern und Larven. Auch die Bestände anderer Fische wurden untersucht. Außerdem wurden zahlreiche Fischereiversuche mit verschiedenen Geräten (Vergleichsfischerei) ausgeführt. Daneben wurden Plankton- und hydrographische Beobachtungen vorgenommen.

Die von Heincke durchgeführten Untersuchungen waren Grundlage für seine im Herbst 1913 und im Frühjahr 1914 vorgelegten praktischen Vorschläge zur Regelung der Seefischerei des nordischen Meeres.

Allerdings wurde die Auswertung durch den Ausbruch des I. Weltkrieges verzögert.

Wie bereits erwähnt, war man bald nach dem I. Weltkrieg zu der Einsicht gekommen, daß Lebensgemeinschaftsforschung mit der Erforschung ihrer einzelnen Glieder gekoppelt werden muß. Hierzu dienten auch die Versuche zur künstlichen Anzucht von Fischen und Wirbellosen. So wurde - wie schon erwähnt - 1924 auf Sylt ein Zweiglaboratorium für Au- 
sternforschung und zur ökologischen Untersuchung des Wattenmeeres eingerichtet. Diese Untersuchungen hatten weiterhin Bezug auf den Vorgang der Landgewinnung. Sie umfaßte die Bedeutung der im Watt lebenden Organismen, wobei der physikalische und der biologische Teil der Anschlickung untersucht wurde.

Bemerkenswert erscheint, da $\beta$ an der Anstalt entgegen einer allgemeinen Tendenz kaum physiologische Arbeiten durchgeführt wurden. Hier stützte man sich lediglich auf Arbeiten von Gästen der Station und bemühte sich etwa ab 1921 um Einrichtung eines physiologischen Laboratoriums. Das Aussetzen der physiologischen Forschung zwischen den beiden Kriegen wurde als Mangel empfunden. Adolf Bückmann behauptete, daß man in der sich stürmisch entwickelnden Fachrichtung selten auf marine Objekte zurückgegriffen habe und eine bedauerliche Entfremdung zwischen Meeresforschung und Physiologie eingetreten sei (vgl. Bückmann 1959). Diese Einschätzung ist angesichts der hervorragenden Arbeiten des deutsch-amerikanischen Physiologen Jacques Loeb ${ }^{145}$, von Otto Meyerhof ${ }^{146}$, Otto Warburg ${ }^{147}$, Hans Driesch ${ }^{148}$ und anderen an marinen Objekten nur bedingt richtig. Hans Winterstein ${ }^{149}$, ab 1911 Direktor des Physiologischen Instituts der Universität Rostock, hatte in einem Gutachten ausführlich die Notwendigkeit der Förderung vergleichender Physiologie niederer Bodentiere begründet. Er betonte, daß sie als Versuchsobjekte für die Medizin von hervorragender Bedeutung seien, z. B. seien Quallen und Schnecken sehr gute Versuchsobjekte zum Studium toxischer Wirkungen.

Für die Einrichtung einer physiologischen Abteilung fehlte es trotz der Bemühungen Heinckes an Möglichkeiten. Erst ab 1938 standen im Neubau physiologische Laboratorien zur Verfügung. Erst nach dem II. Weltkrieg wurde die Physiologie an der Anstalt aufgebaut, des weiteren andere Zweige wie z. B. die Mikrobiologie.

\subsubsection{Die Sicherung ökonomischer und politischer Interessen. Zur Mitarbeit in der Kommission für internationale Meeresforschung}

Auslöser für die Anfertigung des Gutachtens durch den Deutschen Seefischereiverein "Über internationale Untersuchungen der deutschen Meere im Dienst der Seefischerei“ war eine Anfrage der Königlichen Schwedischen Akademie der Wissenschaften an die Uferstaaten von Nord- und Ostsee, wie man zu internationalen Untersuchungen über Hydrographie und Biologie von Ost- und Nordsee und Eismeer stehe.

Der deutsche Seefischereiverein hatte bereits seit 1897 bilaterale Abstimmungen mit einzelnen Staaten vorgenommen. Diese Abstimmungen kamen auf Grund guter Kontakte einzelner Wissenschaftler zustande, so kam es während einer vertraulichen Konferenz in Dortmund vom 19. 3. 1897 mit einem hervorragenden Vertreter der holländischen Seefischerei zu Gesprächen, ebenso mit Vertretern Frankreichs und Großbritanniens. Die Beteiligten befürworteten die Einsetzung einer internationalen Kommission zur wissenschaftlichen Erforschung der Nordsee. Wie im Gutachten ausgeführt wurde, sollte Deutschland, das wichtige Ergebnisse zur Meeresforschung beigetragen hatte, eine internationale Konferenz zwischen Deutschland, den Niederlanden, Belgien, Frankreich, Schweden, Schottland, Norwegen und Dänemark vorschlagen. Von der Tatsache, daß Deutschland die Initiative ergreifen sollte, versprach man sich eine wichtige außenpoliti-

145 Jacques Loeb (1859-1924)

146 Otto Meyerhof (1884-1951)

147 Otto Warburg (1883-1970)

148 Hans Driesch (1867-1941)

149 Hans Winterstein (1879-1963) 
sche Wirkung, es bestand auch die Überzeugung, daß Deutschland diese Rolle aufgrund wissenschaftlicher Leistung zustände.

Deutschland wurde von Karl Brandt, Friedrich Heincke, Oscar Herwig, Hermann Henking und Otto Krümmel vertreten. Nach der Konferenz in Kristiania einigte man sich am 23. 11. 1901 in staatlicher Abstimmung, daß das Reich dem Zentralausschuß für die internationale Meeresforschung beitreten sollte.

Diesem Schritt waren langjährige staatliche Abstimmungsrunden zwischen Auswärtigem Amt, Reichsmarineamt, Reichsschatzamt, Kultusministerium, Landwirtschaftsministerium und dem Ministerium für Inneres vorangegangen. Teilweise wurde auch der Finanzminister hinzugezogen, der bereits 1900 darauf bestand, einen sogenannten „Präzipualbeitrag" in Höhe und Zeit festzulegen, da er schon damals eine „unübersehbare Ausdehnung" der Reichsangelegenheit befürchtete. Diese Sorge sollte sich angesichts des umfangreichen wissenschaftlichen Programmes als berechtigt erweisen. Man einigte sich auf einen Zeitraum von fünf Jahren. Vom Reich sollten für fünf Jahre 120000 Mark getragen werden, von Preußen 30000 M. Auf der 68. Sitzung des Reichstages, die am 15. 3. 1901 stattfand, wurde beschlossen, für die Deutsche Wissenschaftliche Meereskommission ein Büro einzurichten und ein Schiff für Forschungsfahrten zu bauen bzw. zu chartern.

Man kam überein, sowohl in Kiel als auch auf Helgoland Laboratorien für die wissenschaftlichen Meeresuntersuchungen zu installieren. Für Kiel sah man zunächst fünf Planstellen vor, eine für einen Hydrographen, zwei für Zoologen, zwei für Assistenten. An der Anstalt sollten zwei Zoologen (Jahresgehalt je $3000 \mathrm{M}$ ) und ein zoologischer Assistent $(2400 \mathrm{M})$ angestellt werden. Die Anstellungen waren befristet, Ansprüche auf eine etatsmäßige Anstellung im Reichsdienst waren ausgeschlossen.

Wie bei den Ausmaßen des wissenschaftlichen Programms zu erwarten, konnten die Arbeiten $1906 \mathrm{zu}$ keinem Abschluß gebracht werden. Vom Kultusministerium wurden namhafte Wissenschaftler um ein Gutachten gebeten, das die Entscheidung vorbereiten sollte, ob die Arbeiten weitergeführt werden könnten. Gutachter waren u. a. der Leipziger Zoologe Carl Chun, die Akademiemitglieder Ludwig Diels ${ }^{150}$, bis 1914 außerordentlicher Professor in Marburg, später Professor und Direktor des Botanischen Gartens in Berlin, Vahlen und Hensen. Auch Arthur von Auwers, von 1878-1912 Sekretar der Preußischen Akademie der Wissenschaften, wurde um seine Meinung gefragt.

Alle Gelehrten befürworteten selbstverständlich die Weiterführung ernsthaft. Dieses sehr positive Gutachten wurde vom Reichsschatzamt mit großem Argwohn zur Kenntnis genommen. So schrieb man an das Innenministerium:

„Es würde sich also ergeben, daß die Meeresforschung sich zu einer ständigen Wissenschaft ausbilden werde, wie andere von altersher betriebene Wissenschaftszweige. Euerer Excellenz Einverständnis glaube ich darin zu begegnen, daß eine solche Folge bei der Einleitung der Meeresforschung nicht beabsichtigt war" (103).

Man bestand auf kurzfristigen wirtschaftlichen Ergebnissen. Das Reichsschatzamt wollte durchsetzen, die Finanzierung der Arbeiten nur noch auf drei Jahre zu beschränken. Die Wissenschaftler hatten jedoch nicht nur im Kultus- und Landwirtschaftsministerium Verbündete, auch der Reichskanzler selbst und das Auswärtige Amt hielten die Weiterführung für angeraten. Man einigte sich schließlich aber doch auf fünf Jahre. Ab 1910 begannen die heftigen Diskussionen um einen Austritt Deutschlands aus der internationalen Meeresforschung von neuem. Das Reichsschatzamt plädierte wiederum für Austritt (96), die übrigen Institutionen für Weiterführung. Auch der Reichskanzler (95), (109), (110),

150 Ludwig Diels (1874-1945) 
ab 1909 Theobald von Bethmann Hollweg ${ }^{151}$, sprach sich erneut dafür aus, daß Deutschland Mitglied bleibe. Der Vertreter des Auswärtigen Amts machte geltend, daß Deutschland schließlich eine führende Rolle auf dem Gebiet der Fischereizoologie innehabe und der Austritt Deutschlands in wissenschaftlicher und wirtschaftlicher Hinsicht von Schaden sein würde. Das Reichsschatzamt beharrte auf seinem pragmatischen Standpunkt und machte geltend, daß man ursprünglich nur von fünf Jahren ausgegangen war, nun arbeite man schon zehn Jahre und habe lediglich ein einziges praktisches Ergebnis über die Scholle vorgelegt. Dabei sei es allerdings so, daß diese sehr aufwendigen Forschungen die materiellen Interessen der Seefischerei nicht wirklich gefördert hätten, für derartige „uferlosen“ Unternehmen habe man in Deutschland kein Geld mehr.

Auch das Argument des Auswärtigen Amtes, weitere Länder wie Kanada, Frankreich und die USA würden beitreten, interessierte das Reichsschatzamt nicht, der Aufwand würde steigen, weil mit Einzelwünschen zu rechnen sei, die den Aufwand erhöhten:

"Im Gegenteil ist namentlich bei einem Anschlusse der Vereinigten Staaten zu besorgen, daß die zu verfolgenden praktischen Ziele noch in weitere Ferne gerückt werden und der Aufwand sich steigern wird, weil diese Staaten voraussichtlich besondere Wünsche zur Geltung bringen werden, die man schon aus politischer Höflichkeit erfüllen zu müssen glauben wird. M. E. sollte alles vermieden werden, was geeignet wäre, den Beitritt der Vereinigten Staaten zu fördern" (106).

Die Gesamthöhe der Kosten betrage inzwischen immerhin 1160000 Mark, angesichts solcher Summen seien politische Höflichkeiten fehl am Platze. Einer Ausdehnung des Forschungsprogramms für die Scholle auf andere Nutzfische könnte nicht zugestimmt werden.

Glücklicherweise wurde die Meinung des Reichsschatzamtes weder vom Reichskanzler noch von anderen Dienststellen getragen. Die allgemeine Unsicherheit, ob weiterhin Mittel bewilligt werden könnten und die Arbeitsplätze im Labor der Wissenschaftlichen Meereskommission erhalten bleiben könnten, hatte erheblichen Einfluß auf Arbeitsfreude und Engagement der langjährigen Mitarbeiter der Anstalt. So machte Heincke dem Kultusminister am 24. 7. 1909 folgende Mitteilung:

„Die beiden in Betracht kommenden Herren Dr. Reichard und Dr. Franz haben mir erklärt, daß sie sich unter den gegebenen Umständen in ihrer Stellung an der Anstalt nicht bedingungslos bis zum 1. 7. 1910 verpflichten könnten, sondern sich die Möglichkeiten offen halten müßten, eine sich ihnen etwa im Laufe des Jahres auf dem Festlande bietende bessere und sicherere Stelle anzunehmen" (94).

Beide Wissenschaftler schieden 1910 aus der Anstalt aus. Viktor Franz wurde Mitarbeiter des Neurologischen Instituts in Frankfurt/Main. Dort setzte er seine physiologischen Untersuchungen an Sinnesorganen fort. Nebenbei sei bemerkt, daß ihm die aus seiner Sicht eintönigen Arbeiten zur Schollenmarkierung bei Heincke nicht gefielen und er sich auch mit Heincke nicht gut verstand. Die Mittel für die Meeresforschung wurden für weitere vier Jahre bewilligt. Noch während der Reichstagsdebatte vom 3. 2. 1914 (204. Sitzung) kam es zu einer erhitzten Diskussion darüber, ob Deutschland weiter an der internationalen Meeresforschung teilnehmen sollte. Es wurde als unhaltbarer Zustand bezeichnet, daß Wissenschaftler, die seit 13 bis 14 Jahren für die praktische Fischerei arbeiten, nicht fest angestellt werden konnten.

Im Jahre 1913 war Heincke endlich in der Lage, auf Grund seines Generalberichtes über die Scholle den beteiligten Regierungen die ersten auf praktisch-wissenschaftliche

151 Theobald von Bethmann Hollweg (1856-1921) 
Untersuchungen gegründeten Vorschläge zu einem gesetzlichen Mindestmaß für den Fang und den Verkauf von Schollen in der Nordsee vorzulegen. Dieser Vorstoß führte zu Streitigkeiten mit England und Schottland, wo man erst Auswertungen eigener Fangergebnisse abwarten wollte. Es wurde von britischer Seite die Behauptung aufgestellt, daß die Deutsche Bucht die „Kinderstube“ der Scholle sei und das Fischen dort überhaupt verboten werden müßte. Dieses Verbot hätte deutsche Interessen empfindlich gestört. Ein Ausgleich in dem Interessenkonflikt zwischen Deutschland und England wurde schließlich durch vermittelndes Eingreifen Dänemarks herbeigeführt.

Etwa Ende 1913 bis zum Kriegsausbruch verstärkten sich in der Regierung die Diskussionen um die weitere Teilnahme Deutschlands an der internationalen Meeresforschung, zumal Deutschland erhebliche Mitgliedsgebühren zu zahlen hatte und auch die Teilnahme der beiden Mitglieder an den internationalen Konferenzen in Kopenhagen größere Kosten verursachte. Es bleibt zu vermuten, daß finanzielle Probleme der Kriegsvorbereitungen eine Rolle spielten. Wie aus einer geheimen Mitteilung des Staatssekretärs des Reichsmarineamts an das Ministerium des Innern hervorgeht, wurde die Ausdehnung des Reichskriegshafengesetzes auf Helgoland geprüft. Hierbei ging man davon aus, daß Deutschland in einen Seekrieg verwickelt werden würde.

Wegen der Mitgliedschaft Deutschlands in der internationalen wissenschaftlichen Meereskommission (IWK) kam es zu einem umfangreichen Schriftwechsel zwischen Reichskanzlei und Finanzministerium. Der Reichskanzler betonte in einem Schreiben vom 26. 3. 1914 an das Finanzministerium (95), daß die Arbeit nur fortgesetzt werden könne, wenn die materiellen Interessen der Seefischerei im Mittelpunkt ständen. Zwei Monate später gab es zwischen dem Auswärtigen Amt, dem Innenministerium, dem Reichsschatzamt, dem Kultusministerium, dem Finanzministerium und dem Landwirtschaftsministerium eine Abstimmungsrunde, die ergebnislos verlief. Die Verhandlungen wurden durch den I. Weltkrieg unterbrochen, man teilte mit, daß eine weitere Diskussion sich wohl nun erübrige.

Infolge des Seekrieges war eine Befischung der Nordmeere nicht mehr möglich.

Die Zusammenarbeit innerhalb der internationalen Meeresforschung wurde bereits kurz nach Kriegsende neu belebt. 1920 fand im Reichswirtschaftsministerium eine Besprechung statt, an der Vertreter aus nahezu allen Ministerien teilnahmen. Man besprach die Fortsetzung der Arbeiten auf dem Gebiet der internationalen Meeresforschung. Von den Vertretern der Ministerien wurde dieses Anliegen begrüßt, auch wenn sie unterschiedliche Positionen vorbrachten. Heincke und andere Wissenschaftler betonten wissenschaftliche Aspekte und Vorteile. So stellte Heincke heraus, daß der Krieg als unbeabsichtigtes Experiment auf dem Gebiet der Fischwirtschaft betrachtet werden müsse und daß sich dadurch, daß keine Befischung stattfinden konnte, große Fischbestände entwickelt hätten. Es sei jedoch infolge des internationalen Nahrungsbedarfs zu befürchten, daß durch eine zu starke und unkontrollierte Befischung die Bestände geschädigt würden.

Das Auswärtige Amt befürwortete die Belebung aus politischen Gründen, um Deutschlands Stellung wieder zu festigen und der internationalen Isolierung entgegenzuwirken, die Admiralität befürchtete Auseinandersetzungen mit Großbritannien, und das Finanzministerium schlug eine abwartende Haltung vor. Der Vertreter des Auswärtigen Amtes drückte die Hoffnung aus, daß die Wissenschaft zur Wiederannäherung der ehemaligen Feindstaaten beitragen könne. Im Protokoll wurde vermerkt:

„das Gebiet der Wissenschaft und das der Wirtschaft ganz besonders halte er die internationale Meeresforschung dafür geeignet, die erhitzten Gemüter zu beruhigen und Freundschaft, oder zumindestens wieder. korrekte Beziehungen herbeizuführen. Deutsch- 
land besitze wertvolle Einrichtungen in der Anstalt auf Helgoland, einen Dampfer, lange internationale Vorbereitungen in der Sache und dessen werden sich die Feinde Deutschlands und die anderen Teilnehmer der Kommission kaum entraten können" (112).

Heincke unterstützte diese Auffassung und argumentierte, daß der Abbruch der Kontakte vor allem Deutschland träfe, und warnte vor wirtschaftlichen und politischen Nachteilen (112).

Heincke teilte mit, daß neutrale Staaten, selbst England, besser dran seien, und bezeichnete den Krieg als großes Unglück für die internationale Meeresforschung, bedingt durch den internationalen Charakter des Gebietes, des Meeres und ihres Kampfgegenstandes, der Hochseefischerei. In einer 1917 geschriebenen Veröffentlichung führte er aus:

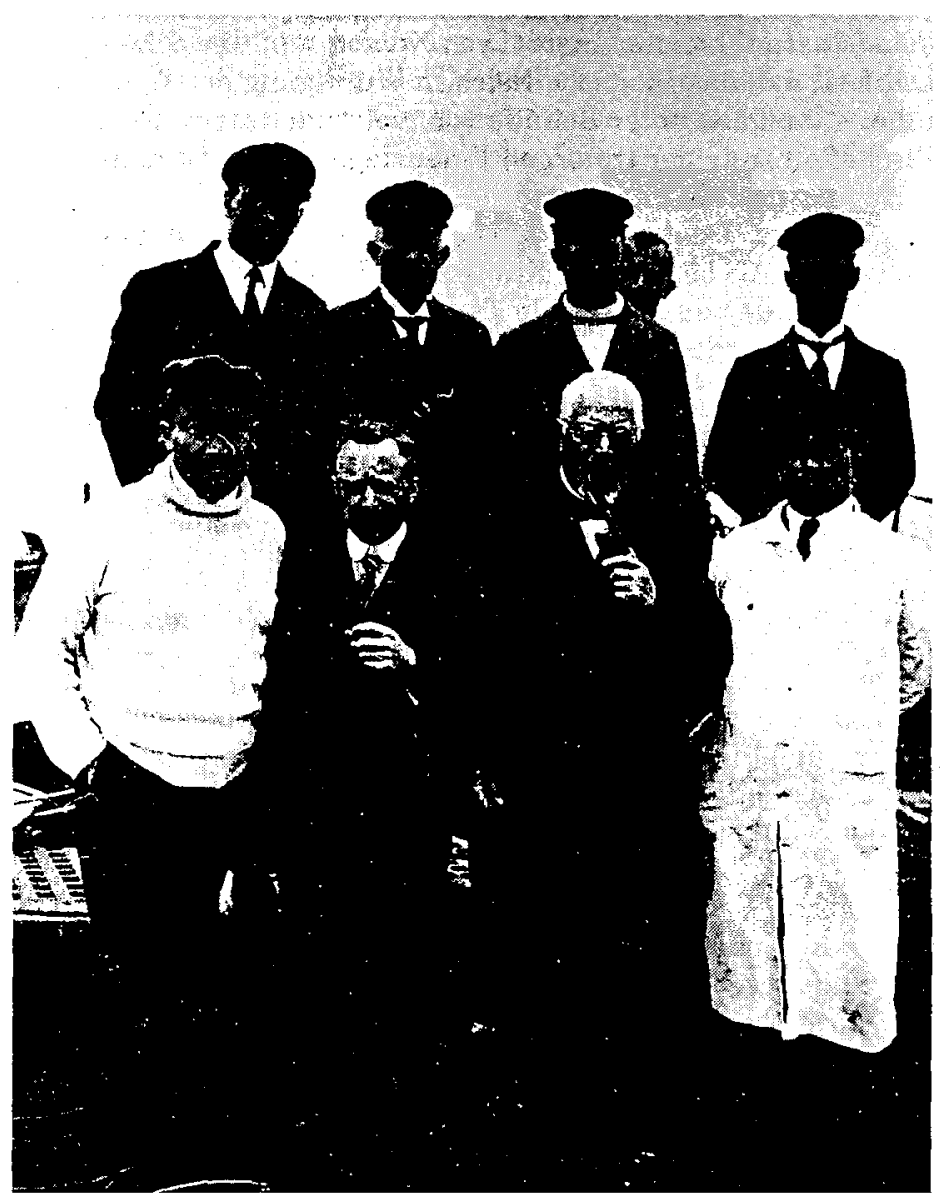

Abb. 39. Einige Mitarbeiter der Biologischen Anstalt auf Helgoland auf dem Forschungsschiff „Poseidon“ 1926. Vorn v. l. n. r. : Prof. Hagmeier, Prof. Schulz, Prof. Strodtmann, Prof. Mielck. Hinten v. l. n. r.: Dr. Zorell, Kpt. Matje, Dr. Heidrich, P. Singer (verd.), Kapit. Hupe. Archiv der Biologischen Anstalt Helgoland. 
„Geht es in diesem Kriege um die Freiheit der Meere, so gewiß auch um die Freiheit der Seefischerei, diese kann nur bedeuten eine auf freies Übereinkommen aller Völker gegründete gemeinsame wirtschaftliche Ausnutzung des Meeres durch einen gesetzlich geregelten vernünftigen Betrieb der Fischerei und durch internationale Fischereibetriebe" (Heincke 1917).

Er formulierte sinngemäß drei rhetorische Fragen, die Deutschland zu beantworten habe, wenn es die Wiederaufnahme prüfe:

Erstens ist die Fischereiwirtschaft notwendig zur Erreichung wichtiger wissenschaftlicher und praktischer Ziele für die Beherrschung des Meeres durch den Menschen und sind die deutschen Leistungen groß genug? Zweitens ist eine Wiederaufnahme der Beziehungen in absehbarer Zeit nach dem Friedensschluß möglich, und drittens hat Deutschland ein besonderes Interesse an ihrer Förderung und Fortsetzung?

Heincke beantwortete alle drei Fragen mit, ja". Er kündigte an, persönlich bei der Wiederaufnahme von Kontakten behilflich zu sein. Seine guten Beziehungen zu Mitgliedern ehemaliger „Feindstaaten“ könnten hierbei von Nutzen sein. Die Sitzungsteilnehmer hielten im Protokoll fest, daß das Deutsche Reich in Würdigung der Wichtigkeit und Unaufschiebbarkeit dieser Aufgabe unter Beihilfe von mehreren internationalen Behörden und Fischereigesellschaften trotz der traurigen Finanzlage die zur Durchführung notwendige Summe zur Verfügung gestellt habe (112).

Doch erst 1926 trat Deutschland wieder in die Internationale Wissenschaftliche Meereskommission ein. Als besonderer Erfolg und als Zeichen für die Anerkennung deutscher Wissenschaft wurde von Mielck die Tatsache bewertet, daß Deutschland nun zwei Kommissionsmitglieder stellte, darunter den Vizepräsidenten. Solchen äußeren Zeichen der Anerkennung wurde nach dem I. Weltkrieg von seiten der Ministerien, insbesondere des Auswärtigen Amtes, große Beachtung geschenkt.

In der Wirtschaftspolitik der Nationalsozialisten kam der Fischerei besondere Bedeutung zu. Carl Heinrici ${ }^{152}$, Staatssekretär a. D. des Innern und Vorsitzender der Deutschen Wissenschaftlichen Kommission für Meeresforschung, betonte in der Einleitung zu den Berichten der DWK der Jahre 1934-37:

„Die deutsche Fischerei hat im Rahmen der neuen Wirtschaftspolitik und des Vierjahresplanes eine erhöhte Bedeutung für die Ernährung des Volkes bekommen. Das gilt im besonderen für die Dampfhochsee- und die große Heringsfischerei, welche die Massenfische für den Großverbrauch anliefern. Der Schiffspark dieser Zweige der Fischerei hat in den letzten Jahren eine durchgreifende Verjüngung und Erweiterung erfahren " (Heinrici $1934-37$ ).

Hagmeier erklärte, daß außer den Mitarbeitern der Arbeitsstelle der Deutschen Wissenschaftlichen Kommission sich die meisten Gelehrten der Anstalt in den Dienst der Fischereiwissenschaft gestellt haben, da die Ergebnisse der wissenschaftlichen Meeresforschung auf dem Wege über die Fischereiforschung der Volksernährung wissenschaftliche Dienste leisten könnten.

Das Reichserziehungsministerium bewilligte zwei Planstellen.

Wie aus den „Anmeldungen der Anstalt für den Staatshaushaltsplan 1936“ hervorgeht, wurden aber auch die Untersuchungen im Watt wissenschaftlich, volkswirtschaftlich und strategisch begründet (117). Ziel der Begründungen war es, eine zusätzliche Planstelle zu erhalten. Es wurden folgende Argumente ins Feld geführt:

152 Carl Heinrici (1876-1944) 
1. die fischereiliche Ausnutzung des Wattenmeeres und die Gewinnung von verschiedenen Rohstoffen könne durch wissenschaftliche Untersuchungen gefördert werden,

2. bei den Landgewinnungsarbeiten habe sich als wichtig herausgestellt, die aufbauende und den Boden verbessernde Tätigkeit einer ganzen Anzahl von Meeresorganismen kennenzulernen und die natürlichen Bedingungen festzustellen, unter denen sie gedeihen können,

3. bei den Arbeiten der Strom- und Fahrwasserregulierung spielen die Organismen ebenfalls eine Rolle.

Es wurde darauf verwiesen, daß die Kriegsmarine, d. h. das Strombauressort der Marinewerft Wilhelmshafen, an den biologischen Arbeiten im Jadebusen besonders interessiert sei und Bereitschaft zeige, diese Forschungen zu unterstützen. In Wilhelmshafen befand sich ein großer Hafen der Kriegsmarine. Ein weiteres Argument war jedoch, wie seit 1892, die Notwendigkeit der Erforschung deutscher Heimat als kultureller Wert und die Befürchtung, in diesem Punkte hinter anderen Nationen zurückzustehen. So hieß es sinngemäß, $\mathrm{da} B$ ein großes biologisches Interesse an den Untersuchungen im Wattenmeer bestände, denn es sei beschämend, wie wenig die Deutschen bisher in die Erforschung eigener Küstengebiete eingedrungen seien im Gegensatz zu den übrigen Nordseeanliegerstaaten.

\subsection{Die Fortführung ornithologischer Traditionen auf Helgoland}

Im Jahre 1909 wurde Hugo Weigold als wissenschaftlicher Assistent für Meeresbiologie eingestellt. Seine Liebe gehörte jedoch von Anfang an der Ornithologie. Er war es, der nicht nur mit Duldung, sondern großer Förderung Friedrich Heinckes die ornithologische Forschung auf Helgoland aufbaute. Weigolds Wirken ist ein Beispiel dafür, wie Einzelwissenschaftler durch ihr Engagement Einfluß auf die Gestaltung des Forschungsprogrammes nahmen. Ab 1910 arbeitete Weigold hauptamtlich auf dem Gebiet der Ornithologie. Der Wissenschaftler knüpfte an die langjährige Tradition der Vogelkunde auf Helgoland an, u. a. an Arbeiten des Kunstmalers Heinrich Gätke. Gätke, das sei nebenbei erwähnt, war ein weitläufiger Verwandter Theodor Fontanes ${ }^{153}$. Er galt als schöner Mann und wurde von der Schriftstellerin Fanny Lewald ${ }^{154}$ mit dem Künstler Benvenuto Cellini ${ }^{155}$ verglichen, der als Abenteurer und Frauenheld bekannt war (vgl. Fontane 1907).

Gätke knüpfte an eine lange Tradition der Vogelforschung auf Helgoland an, die zumeist von interessierten Naturwissenschaftlern bzw. Laien betrieben wurde.

Unter dem Einfluß der um 1800 erschienenen ornithologischen Bestimmungsbücher entwickelte sich in Deutschland vielerorts die Lust zum Vogelsammeln. So enstand in Bremen ein ornithologisches Museum, an dessen Ausbau ein dort beschäftigter Präparator, ein sogenannter „Ausstopfer“, großen Anteil hatte. Von ihm erlernte der Helgoländer Schuhmacher Erich Koopmann ${ }^{156}$ die Kunst des Präparierens.

Auf Koopmann geht ein wesentlicher Teil der Helgoländer Vogelsammlung zurück. Auch der außerordentliche Professor für Mineralogie Friedrich B. Hoffmann ${ }^{157}$ widmete sich auf Helgoland ornithologischen Beobachtungen, die in wissenschaftliche Veröffentlichungen eingingen. Er erkannte, daß das vielfältige Vogelleben auf Helgoland dem

153 Theodor Fontane (1819-1898)

154 Fanny Lewald (1811-1889)

155 Benvenuto Cellini (1500-1571)

156 Erich Koopmann (gest. 1835)

157 Friedrich B. Hoffmann (1797-1836) 
Ornithologen große Möglichkeiten bietet. Auch der "Vater"s der deutschen Ornithologie, Johann F. Naumann ${ }^{158}$, Professor und Inspektor des Ornithologischen Museums des Herzogs von Anhalt-Köthen, fand auf Helgoland Anregungen. Seine zwölfbändige „Naturgeschichte der Vögel Deutschlands“", die von späteren Autoren bearbeitet und weitergeführt wurde, gehört zu den ornithologischen Standardwerken. Ihm zu Ehren nannte die Deutsche Ornithologische Gesellschaft ihr Publikationsorgan „Naumannia“.

In folgender Tabelle sind wichtige Daten über die Vogelforschung auf Helgoland im 19. Jahrhundert zusammengefaßt:

Tabelle 2. Ornithologische Traditionen auf Helgoland

1822 Erich Ernst Koopmann (Helgoland) übergab dem Zoologisehen Museum in Berlin eine auf Helgoland zusammengetragene „Naturaliensammlung“, die $u$. a. Vogelbälge enthielt.

1824 Der Geologe Friedrich B. Hoffmann veröffentlichte eine ausführliche Abhandlung über naturwissenschaftliche Ergebnisse seiner Reise nach Helgoland, in der er auch auf seine ornithologischen Beobachtungen einging.

1837 Der Kunstmaler und Amateurforscher Heinrich Gätke trug auf Helgoland eine der bedeutendsten Balgsammlungen einheimischer und fremder Vögel zusammen.

1840 Johann Friedrich Naumann fand wichtige Anregungen für sein Buch „Naturgeschichte der Vögel Deutschlands“, u. a. lernte er die Vogelsammlung von Carsten Peter Reimers $^{159}$ (Barbier und Chirurg) kennen und schätzen.

Weigold bearbeitete die Sammlung Gätkes, revidierte den Bestand an Bälgen, ordnete, etikettierte und katalogisierte ihn. Dann nahm er die von Gätke begonnenen regelmäßigen und systematischen Beobachtungen des Vogelzuges wieder auf. Thm gelang die Erbeutung kostbarer Unikate, und die Helgoländer Sammlung erreichte Ende 1910 bereits 408 Bälge mit 150 Arten. In Abstimmung mit der Vogelwarte Rossitten wurde damit begonnen, mit Ringen markierte Vögel auf Helgoland auszusetzen. In der Sapskuhle, einer Geländevertiefung auf dem Oberland, wurde ein Fanggarten eingerichtet, der später zum Versuchsgarten ausgebaut wurde.

Am Anfang standen phänomenologische Beobachtungen, die Weigold in einem Vogelzugkalender niederlegte. Bald kam die Vogelberingung als wichtiges Mittel für die Erforschung des Vogelzuges hinzu. Daran schlossen sich Forschungen an über Ursachen und Begleitumstände des Zuges wie Licht, Wetter, Jahreszeiten, aber auch die inneren Ursachen, die den Zugtrieb auslösen. Diese wurden z. T. mittels physiologischer Untersuchungen geprüft. Mit Hilfe der Beringung von Vögeln konnten wertvolle Erkenntnisse gewonnen werden, so wurden bei verschiedenen Arten Alters- und Geschlechtsunterschiede erforscht und auch verschiedene Rassen und Formen von Helgoländer Zugvögeln bestimmt. Besondere Aufmerksamkeit widmete man den Seevögeln, deren Brutkolonien und namentlich auch der Trottellumme (Uria aalge), die ja auf Helgoland ihren Brutplatz hat.

Neben den natürlichen Voraussetzungen, der Konzentration des Vogelzuges auf der Hochseeinsel und der Insel als Brutplatz gab es mehrere Gründe für die Förderung der Ornithologie auf Helgoland:

158 Johann Naumann (1780-1857)

159 Carsten Reimers (geb. 1805) 
1. das große Engagement Hugo Weigolds und seine Fähigkeit, amtliche Stellen zur Unterstützung zu gewinnen, die sich $\mathbf{u}$. a. in den zahlreichen Anträgen auf finanzielle Unterstützung und in geschickt verfaßten Berichten widerspiegelte,

2. die Verknüpfung der Vogelforschung mit dem sich in Deutschland entwickelnden Naturschutzgedanken, der die Bewahrung gerade der deutschen Natur als kulturellen Wertbegriff,

3. die propagandistische Bedeutung der Beringungstätigkeit unter Einbeziehung breitester Bevölkerungskreise, verbunden mit dem Bestreben, einen vermeintlichen oder echten Vorsprung gegenüber anderen Ländern sichern zu wollen.

Über seine Helgoländer Beobachtungen hinaus nutzte Weigold lange Reisen, um im Ausland seine Studien fortzusetzen. Sein Engagement ging so weit, daß er an jahrelangen privat ausgestatteten Forschungsexpeditionen $u$. a. nach Tibet und China teilnahm und sogar sein Leben aufs Spiel setzte. Seine Forschungsberichte, besonders der über seine fünfjährige Forschungstätigkeit während des I. Weltkrieges in China, zeugen von der hohen „Berichtskunst" des Verfassers. Sie waren dazu geeignet, das Ministerium von seiner Tüchtigkeit zu überzeugen:

„Ich folgte den Wandervögeln auf ihren Wegen, wurde selbst zum Wandervogel, um sie verstehen zu lernen, und tummelte mich im Flugzeug unter ihnen, um mit ihren Augen sehen zu lernen. Denn gab nicht vielleicht der eine Fleck Helgoland ein falsches Bild? Um das zu prüfen, sah ich den Wanderzug an mir vorüberrauschen in Ungarn und an der Küste Mesopotamiens, an den Küsten des Atlantiks und des Mittelmeeres und nun in allen Ecken des gewaltigen China ... Vor allem fesselnd ist die Frage, ob man aus dem übergrossen Reichtum der Arten von so vielen Tier- und Pflanzenfamilien schliessen darf, dass hier eins der grössten Entwicklungszentren der Erde liegt. Und dann die gesamte Phänologie“ (69)!

Weigold stellte die Vogelforschung auf Helgoland, nach Aussage seines Direktors, auf eine neue Grundlage und organisierte die Arbeit nach dem Vorbild der Vogelwarte Rossitten. In mehreren sehr beachteten Veröffentlichungen war es Weigold gelungen, auf die Bedeutung Helgolands als Durchzugsgebiet für seltene Vögel hinzuweisen (vgl. Weigold 1910).

Der enthusiastische Gelehrte genoß nicht nur die Unterstützung seines Direktors (148), sondern auch der Deutschen Ornithologischen Gesellschaft, namhafter Ornithologen Deutschlands und Österreichs sowie der Staatlichen Dienststelle zur Erhaltung der Naturdenkmäler in Preußen (66) (vgl. Tschusi zu Schmidhofen 1920). Auch die Preußische Akademie der Wissenschaften konnte Weigold für die finanzielle Förderung seiner Arbeiten gewinnen, wobei ihm Heincke mit Gutachten zur Seite stand, die bereits 1912 die Bedeutung des Forschungsgegenstandes und die Persönlichkeit des Bearbeiters ins rechte Licht rückten (70).

Die Bedeutung Helgolands für den Artenschutz konnte von Heincke und seinem Mitarbeiter bereits ab 1908 gegenüber staatlichen Stellen verteidigt werden. Anlaß war der Ausbau Helgolands zur Festung. Er sah seine Aufgabe darin, die Beeinträchtigung als Raststätte der Wandervögel und eines Naturdenkmales auf ein möglichst geringes Maß zu beschränken. Heincke verwies darauf, daß die von Jahr zu Jahr an Umfang zunehmende Bebauung des Oberlandes mit Festigungsanlagen, der Bau des neuen Marinehafens, die Herstellung von Schutzmauern vor der Felsenwand der Westseite u. a. den Helgoland passierenden Zugvögeln die Gelegenheiten zum Rasten auf der Insel mehr und mehr verringerten und damit auch die Beobachtung ihrer Wanderzüge erschwerten, ja sogar die berühmte Lummenkolonie der Insel in ihrem Bestande bedrohten.

Die Mitarbeiter der Anstalt konnten erreichen, daß die Kriegsmarine Rücksicht auf 
das berühmte Naturdenkmal nahm und die im Bau begriffene Schutzmauer nicht am Lummenfelsen vorbeiführte, was das sichere Eingehen der Kolonie bedeutet hätte, sondern beiderseits dicht am Lummenfelsen abschließen ließen, so daß dieser ins Meer hinausragte und den jungen Lummen den ungefährdeten Absprung ins Wasser gestattete.

Die Arbeiten waren im wesentlichen dreierlei: die regelmäßige, beinahe tägliche Beobachtung der Vogelwelt auf Insel und Düne. Während der Zugzeit wurde sie auch nachts durchgeführt. Zweitens wurden durch parallele meteorologische Beobachtungen Zusammenhänge zwischen Vogelzug und Witterung hergestellt. Drittens wurden Beringungsversuche angestellt, um Wanderstraßen der Zugvögel festzustellen. So wurden zwischen 1910 und 1913 mehr als 10000 Vögel beringt. Hierbei konnte sich die Anstalt auf viele freiwillige Helfer stützen. Die Vogelberingung erhielt nach dem I. Weltkrieg besonderes politisches Gewicht. Vogelzugforschung war damals in Mode gekommen, und zahlreiche Menschen wandten sich mit Fragen und Problemen an die Vogelwarte. So verwies Mielck in einem Schreiben an das Kultusministerium auf das

„ständig wachsende Interesse bei den Wissenschaftlern und im Volke für die Vogelkunde schlechthin und den Naturschutz und Vogelzugsforschung insbesondere" (73).

Nach Beendigung des I. Weltkrieges waren keine Mittel mehr für Reisen und Veröffentlichungen vorhanden, und Weigold hatte sogar Schwierigkeiten, einen von ihm verfaßten Vogelzugatlas zu publizieren. Er beantragte bei der Notgemeinschaft Deutscher Wissenschaft einen Zuschuß für die Fertigstellung seiner Arbeit. Seine Bitte wurde 1921 zunächst mit folgender Begründung abgelehnt:

„.. wird sie (die Notgemeinschaft deutscher Wissenschaft, d. V.) ihre Unterstützung nur dann gewähren können, wenn 1. die abzuschließende Arbeit von überragender Bedeutung für die Wissenschaft ist, oder 2. wenn durch Verzögerung des Abschlusses die Gefahr besteht, das andere, z. B. Ausländer, dem Autor zuvorkommen. Weder das eine noch das andere kommt in Betracht. Eine andere Frage wäre die Bewilligung eines Zuschusses zum Druck der fertigen Arbeit, aber das ist cura posterior" (72).

Sein Direktor Mielck setzte sich beim Landwirtschaftsministerium für die Bewilligung von Geldern für die Fortführung der Arbeiten auf der Vogelwarte und für die Fertigstellung des Atlas' ein. Das Geld wurde bewilligt. Weigold war es trotz seiner wissenschaftlichen Erfolge nicht gelungen, eine Kustodenstelle zu bekommen. Die Diskussionen zogen sich über Jahre hin. Obwohl der engagierte Ornithologe von mehreren Seiten Unterstützung erhielt, war er nicht erfolgreich mit seinen Bemühungen (70), (71). Er verließ die Insel, um als Leiter eines Provinzialmuseums auf dem Festlande den Naturschutzgedanken zu verbreiten, wie er das Kultusministerium wissen ließ (vgl. I. Weigold 1986). Seine Arbeit wurde ab 1924 von Rudolf Drost weitergeführt.

Mielck bemühte sich stets um Gelder für die ornithologische Forschung. Hierbei bewies er große Hartnäckigkeit. Zunächst versuchte er, das Landwirtschaftsministerium um finanzielle Unterstützung zu bitten, und argumentierte, daß Vögel schließlich für die Landwirtschaft von besonderem Interesse seien. Leider konnte Mielck die Verantwortlichen im Landwirtschaftsministerium nicht überzeugen. Er wandte sich nunmehr an das Kultusministerium und hob zwei Aspekte hervor.

Erstens appellierte er an die Interessen Preußens und betonte, daß die Wissenschaftler des In- und Auslandes und weite Kreise des Volkes an die Vogelwarte Helgoland die gleichen Anforderungen stellten wie an die Vogelwarte Rossitten (in Ostpreußen).

Zweitens müsse die führende Stellung der deutschen Wissenschaft auf dem Gebiet der Vogelberingung erhalten und verteidigt werden, zumal jetzt nach deutschem Vorbild und dem skandinavischer Länder alle europäischen Staaten und die USA damit begonnen hätten und diese eifrig betrieben. 
Mielck berief sich darauf, daß allein im Jahre 1928 schon 2000 Briefe aus der Bevölkerung eingetroffen seien, 1931 sogar 5500. Er hob hervor, daß die Vogelwarte mit zahlreichen Vereinen und Schulen und mit weiten Volkskreisen, die mitarbeiteten und sich Auskunft und Rat holten, in Verbindung stände. Als Beringungszentrale habe sie mehr als 500 Mitarbeiter in allen deutschen Landen (73). Diese Begründung half, ab 1930 Geld vom Reichsernährungsministerium und dem Ministerium für Wissenschaft, Kunst und Volksbildung zu erhalten.

Obwohl sich mit dem Jahre 1933 die Bedingungen für Wissenschaftler zunächst stark verschlechterten, was u. a. in der im März 1933 vom Finanzminister erlassenen Reisekostenbestimmung zum Ausdruck kam, die festlegte, daß Reisen zu Kongressen und anderen wissenschaftlichen Veranstaltungen von den betreffenden Beamten selbst zu tragen seien, wurde die Vogelzugforschung auf Helgoland weiter gefördert. Arthur Hagmeier, inzwischen neuer Direktor der Anstalt, verstand es mit viel Geschick, durch zeitgemäße Argumentation weitere Mittel für die Vogelzugforschung einzuwerben:

„Aufklärung und Werbung weitester Volkskreise, besonders auf dem Wege über die Beringung. Ferner werden Führungen und Kurse wissenschaftlicher Art abgehalten. Der Volksbildung dient die Vogelwarte durch Vorträge, Museum, Herstellung und Verleih von Lauf- und Lichtbildern weiter Volkskreise durch Schriftwechsel und Veröffentlichungen. Viele Volksgenossen sehen in der Vogelwarte eine Stelle, bei der man sich über Fragen der Vogelkunde und des Naturschutzes Auskunft holen kann" (75) .

Einen Grund dafür, daß diese Argumentation erfolgreich sein konnte, ist sicher in der veränderten Rolle zu suchen, die die Biologie zu Beginn der dreißiger Jahre zu spielen begann. So war ab 1931 Biologie Pflichtfach im Schulunterricht geworden. Dem Fach wurde vor dem Hintergrund eines neuen Verhältnisses zur Natur, wie es u. a. in der besonderen Förderung der ärztlichen Naturheilkunde durch den nationalsozialistischen Staat zum Ausdruck kam (vgl. Thom und Caregorodav 1989), oder auch in der Verabschiedung eines Tierschutz- bzw. Naturschutzgesetzes.

Dem Biologen kam eine besonders wichtige Aufgabe bei der Vermittlung von Kenntnissen an breite Kreise der Bevölkerung zu. Der Vogelschutz war hierbei von besonderer Bedeutung. Die Vogelwarte auf Helgoland galt als Symbol deutscher Wissenschaft. Wurde im Ausland ein Vogel gefunden, der auf Helgoland beringt worden war, beschäftigte dieser Vorfall Diplomaten. So schickte die deutsche Gesandtschaft aus Addis Abeba am 22. 9. 1933 an das Auswärtige Amt folgende Note:

„Ein weiterer aus Deutschland gekommener Vogel ist in der Nähe von Gondar gefangen worden, seine hier beigefügte Erkennungsmarke hat mir die Regierung mit einer Note vom gestrigen Tage zugestellt. Die Landung der beiden Vögel hier hat einen tiefgehenden Eindruck gemacht" ${ }^{\text {(74). }}$

$\mathrm{Ab} 1940$ wurden die Arbeiten der Station durch Einberufungen gestört, wie es offiziell hieß, später durch Luftangriffe. Während des Krieges war die Zahl der Mitarbeiter immer kleiner geworden. Ab 1941 befanden sich außer dem Leiter der Vogelwarte, Drost, nur noch einige weibliche Bürokräfte in der Station. Trotzdem ist es erstaunlich, daß Drost noch im Sommer 1943 in der Zeitschrift „Der Biologe“ über neue Forschungsergebnisse berichtete. Der Forscher nutzte die Kriegssituation und die Tatsache, daß der Leuchtturm ausgeschaltet war, zur Untersuchung der Frage, welche Auswirkung der Leuchtturm auf den Vogelzug hat. Drost bezeichnete diese Tatsache als "großes Experiment“ (Drost 1943). Er stellte u. a. fest, daß die berühmten Helgoländer Massenzugtage trotz ausgeschaltetem Leuchtturm nicht ausblieben. Ein Teilergebnis war auch die Aufklärung des Zusammenhanges zwischen meteorologischen Erscheinungen und dem Vogelzug. Auch die Beringungsarbeit wurde fortgesetzt, obwohl keine Freiwilligen mehr zur Verfügung standen. Mit der Evakuierung aller Einwohner im Jahre 1945 fanden alle Arbeiten ein jähes Ende. 